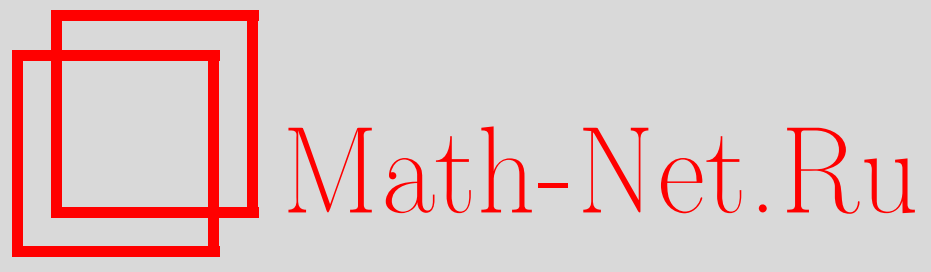

Р. Р. Гонцов, В. А. Побережный, Г. Ф. Хельминк, Деформации систем линейных дифференциальных уравнений, УМН, 2011, том 66, выпуск 1, 65-110

DOI: https://doi.org/10.4213/rm9404

Использование Общероссийского математического портала Math-Net.Ru подразумевает, что вы прочитали и согласны с пользовательским соглашением http://www.mathnet.ru/rus/agreement

Параметры загрузки:

IP : 3.85 .7 .115

26 апреля 2023 г., 12:41:33

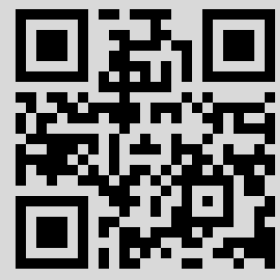




\title{
Деформации систем линейных дифференциальных уравнений
}

\author{
Р. Р. Гонцов, В. А. Побережный, Г. Ф. Хельминк
}

\begin{abstract}
Статья посвящена деформациям систем линейных дифференциальных уравнений с мероморфными коэффициентами. Рассматриваются вопросы существования и классификации деформаций, приводится анализ глобального и локального поведения решений уравнений деформаций в окрестности их особого множества. Некоторые классические результаты, касающиеся изомонодромных деформаций фуксовых систем, обобщаются на случай интегрируемых деформаций мероморфных систем.

Библиография: 40 названий.
\end{abstract}

Ключевые слова: голоморфное расслоение, мероморфная связность, интегрируемость, монодромия, свойство Пенлеве, изомонодромная деформация.

\section{СОДЕРЖАНИЕ}

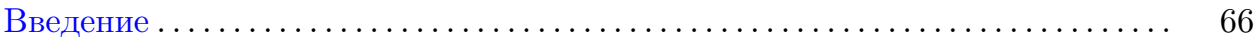

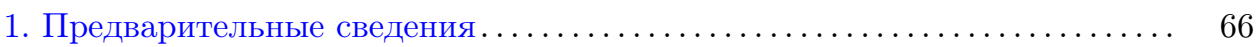

1.1. Линейные системы и монодромия..................... 66

1.2. Фуксовы системы: локальная и глобальная теория ........... 68

1.3. Интегрируемость пфаффовых систем.................. 71

2. Интегрируемые деформации мероморфных связностей ............. 74

3. Изомонодромные деформации фуксовых систем................. 80

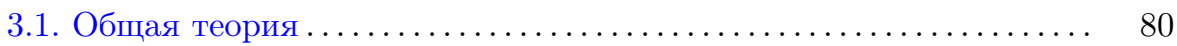

3.2. Изомонодромные деформации Шлезингера................. 83

3.3. Резонансные изомонодромные деформации .............. 85

4. Интегрируемые деформации и свойство Пенлеве ................. 89

4.1. Фредгольмовы операторы ....................... 89

4.2. Нетривиальность деформируемого расслоения ............ 92

4.3. Мероморфное продолжение ...................... 97

4.4. Приложение к системам Гарнье....................... 102

Работа выполнена при поддержке программы "Ведущие научные школы" (грант НШ-8508.2010.1), РФФИ (грант № 08-01-00667, 11-01-00339) и программы NWO "Geometric Aspects of Quantum Theory and Integrable Systems" (грант № 047.017.015).

(C) Р. Р. Гонцов, В. А. ПоБЕРЕЖный, Г. Ф. ХЕльминк, 2011 


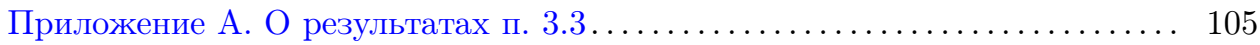

Приложение В. Симплектические структуры................... 107

Список литературы ................................ 108

\section{Введение}

Статья устроена следующим образом. В первом, вводном, разделе мы напоминаем некоторые стандартные понятия, широко используемые при исследованиях мероморфных и, в особенности, фуксовых линейных систем. Это понятие монодромии, основы теории Левеля, а также краткое введение в интегрируемость пфаффовых систем, которые появляются на протяжении всего текста. Особое внимание уделено резонансным особым точкам фуксовых систем и их значимости в вопросах единственности.

Второй раздел посвящен интегрируемым деформациям мероморфных связностей. Мы приводим доказательство существования такой деформации для любого исходного голоморфного векторного расслоения над сферой Римана $\overline{\mathbb{C}}$, снабженного мероморфной связностью.

В третьем разделе изучаются изомонодромные деформации фуксовых систем, или связностей с особенностями в виде полюсов только первого порядка. Такие деформации являются частным случаем интегрируемых. Тот факт, что любая изомонодромная деформация естественным образом связана с некоторой матричной дифференциальной 1-формой, обладающей некоторыми специальными свойствами, позволяет полностью классифицировать изомонодромные деформации фуксовых систем, начиная с простейшего случая деформации Шлезингера и заканчивая наиболее общей резонансной деформацией с дополнительными параметрами. Эта классификация имеет довольно ясную геометрическую интерпретацию в терминах резонансов и их динамики, что обсуждается в приложении А.

В четвертом разделе исследуются свойства интегрируемых деформаций. Представив основные методы теории Фредгольма, мы изучаем (не-)тривиальность деформируемого расслоения, свойства тета-дивизора, мероморфное продолжение и свойство Пенлеве на более общем уровне интегрируемых деформаций, получая аналоги классических результатов, касающихся изомонодромных деформаций фуксовых систем. В заключение мы применяем результаты о поведении изомонодромных семейств Шлезингера в окрестности тета-дивизора к оценке порядков подвижных полюсов интегрируемых систем Гарнье. Приложение В повествует о симплектической составляющей изомонодромных деформаций.

\section{1. Предварительные сведения}

1.1. Линейные системы и монодромия. Рассмотрим мероморбную линейную систему на сфере Римана $\overline{\mathbb{C}}$, т. е. систему $p$ линейных обыкновенных дифференциальных уравнений с особыми точками $a_{1}^{0}, \ldots, a_{n}^{0} \in \mathbb{C}$ и, возмож- 
но, $\infty$. Такая система может быть записана в виде

$$
\frac{d y}{d z}=B(z) y, \quad B(z)=\sum_{i=1}^{n} \sum_{l \geqslant 1} \frac{B_{i l}^{0}}{\left(z-a_{i}^{0}\right)^{l}}+\sum_{l \geqslant 0} B_{\infty l}^{0} z^{l}
$$

где $y(z) \in \mathbb{C}^{p}$ и $B_{i l}^{0}, B_{\infty l}^{0}-$ матрицы размера $p \times p$, равные нулю при достаточно больших $l$. Конформным преобразованием $z \mapsto \frac{a z+b}{c z+d}$ всегда можно добиться того, чтобы все особенности располагались в конечной комплексной плоскости. Это значит, что система (1) может быть сведена к такой, у которой все матрицы $B_{\infty l}^{0}$ равны нулю и

$$
\sum_{i=1}^{n} B_{i 1}^{0}=0
$$

чем обеспечивается отсутствие $\infty$ среди особых точек.

Систему (1) также можно интерпретировать в более геометрических терминах следующим образом. Рассмотрим открытое множество $U=\overline{\mathbb{C}} \backslash\left\{a_{1}^{0}, \ldots, a_{n}^{0}\right\}$ и тривиальное векторное расслоение $E^{0}=U \times \mathbb{C}^{p}$ ранга $p$ над $U$ с базисом сечений $s_{1}, \ldots, s_{p}$, определяемых соотношениями $s_{i}(z)=e_{i}$ для всех $z \in U$. Здесь $e_{i}$ обозначает стандартный $i$-й базисный вектор пространства $\mathbb{C}^{p}$. В расслоении $E^{0}$ имеется голоморфная связность

$$
\nabla^{0}: \Gamma^{0}\left(E^{0}\right) \rightarrow \Gamma^{1}\left(E^{0}\right)
$$

- линейное отображение из пространства $\Gamma^{0}\left(E^{0}\right)$ голоморфных сечений расслоения $E^{0}$ в пространство $\Gamma^{1}\left(E^{0}\right)$ голоморфных дифференциальных 1-форм на $U$ со значениями в $E^{0}$, удовлетворяющее соотношению

$$
\nabla^{0}(f s)=s d f+f \nabla^{0}(s)
$$

где $s \in \Gamma^{0}\left(E^{0}\right)$ и $f$ - голоморфная в $U$ функция. Данная связность определяется формой $\omega^{0}=-B(z) d z$ в базисе $\left\{s_{i}\right\}$ - это значит, что $\left(s_{1}, \ldots, s_{p}\right)$ отображается в $\left(s_{1}, \ldots, s_{p}\right) \omega^{0}$ под действием $\nabla^{0}$. Для малого диска $D \subset U$ локальная голоморфная вектор-функция $y=\sum_{i=1}^{p} y_{i} s_{i}$ из уравнения $(1)$, рассмотренного в $D$, соответствует при этом сечению расслоения $\left.E^{0}\right|_{D}$. Такие сечения называются горизонталъными, поскольку они удовлетворяют соотношению $\nabla^{0}(y)=0$.

Важной характеристикой мероморфной линейной системы (или голоморфной связности в тривиальном векторном расслоении над $U$ ) является монодромия, или представление монодромии, определяемое ниже. Предполагая опять, что особенности $a_{1}^{0}, \ldots, a_{n}^{0}$ расположены в конечной комплексной плоскости, рассмотрим в окрестности $D$ неособой точки $z_{0}$ росток фундаментальной матрицы $Y(z)$ системы (1). Если снова использовать геометрические термины, то можно сказать, что столбцы матрицы $Y(z)$ образуют базис в пространстве горизонтальных (по отношению к $\nabla^{0}$ ) сечений расслоения $\left.E^{0}\right|_{D}$. Аналитическое продолжение матрицы $Y(z)$ вдоль произвольной петли $\gamma$, начинающейся в $z_{0}$ и лежащей в $\overline{\mathbb{C}} \backslash\left\{a_{1}^{0}, \ldots, a_{n}^{0}\right\}$, преобразует эту матрицу в, вообще говоря, 
другую матрицу $\widetilde{Y}(z)$. Две фундаментальные матрицы связаны с помощью невырожденной матрицы перехода $G_{\gamma}$, соответствующей петле $\gamma$ :

$$
\tilde{Y}(z)=Y(z) G_{\gamma}
$$

Отображение $[\gamma] \mapsto G_{\gamma}^{-1}$ зависит только от гомотопического класса $[\gamma]$ петли $\gamma$ и, таким образом, определяет представление

$$
\chi: \pi_{1}\left(\overline{\mathbb{C}} \backslash\left\{a_{1}^{0}, \ldots, a_{n}^{0}\right\}, z_{0}\right) \rightarrow \mathrm{GL}(p, \mathbb{C})
$$

фундаментальной группы пространства $\overline{\mathbb{C}} \backslash\left\{a_{1}^{0}, \ldots, a_{n}^{0}\right\}$ в пространство невырожденных комплексных матриц размера $p \times p$. Это представление называется монодромией линейной системы (или связности $\nabla^{0}$ ).

Матрица монодромии системы (1) в особой точке $a_{i}^{0}$ по отношению к фундаментальной матрице $Y(z)$ - это матрица $G_{i}$, соответствующая простой петле $\gamma_{i}$, обходящей точку $a_{i}^{0}$, так что $G_{i}^{-1}=\chi\left(\left[\gamma_{i}\right]\right)$. Матрицы $G_{1}, \ldots, G_{n}$ являются образующими группы монодромии системы (1). Они удовлетворяют условию $G_{1} \cdots G_{n}=\mathrm{Id}$, следующему из соотношения $\gamma_{n} \cdots \gamma_{1}=e$ на образующие фундаментальной группы, упорядоченные подходящим образом.

Если взять другую фундаментальную матрицу $Y^{\prime}(z)=Y(z) C, C \in \mathrm{GL}(p, \mathbb{C})$, системы (1), то соответствующими матрицами монодромии будут $G_{i}^{\prime}=C^{-1} G_{i} C$. Подобным образом матрицы $G_{i}$ зависят и от выбора начальной точки $z_{0}$. Следовательно, монодромия линейной системы определена с точностью до общего сопряжения на постоянную невырожденную матрицу, и правильнее говорить, что монодромия является элементом пространства классов сопряженности $p$-мерных представлений группы $\pi_{1}\left(\overline{\mathbb{C}} \backslash\left\{a_{1}^{0}, \ldots, a_{n}^{0}\right\}\right)$, которое мы обозначим через

$$
\mathscr{M}_{a^{0}}=\operatorname{Hom}\left(\pi_{1}\left(\overline{\mathbb{C}} \backslash\left\{a_{1}^{0}, \ldots, a_{n}^{0}\right\}\right), \operatorname{GL}(p, \mathbb{C})\right) / \operatorname{GL}(p, \mathbb{C}) .
$$

Завершим данный пункт следующим наблюдением. Имея голоморфную связность в голоморфно тривиальном векторном расслоении ранга $p$ над $U=$ $\overline{\mathbb{C}} \backslash\left\{a_{1}^{0}, \ldots, a_{n}^{0}\right\}$, мы определяем ее монодромию как $p$-мерное представление группы $\pi_{1}(U)$. Обратное также имеет место: по произвольному $p$-мерному представлению $\chi$ группы $\pi_{1}(U)$ можно однозначно построить голоморфное векторное расслоение ранга $p$ над $U$ (которое тривиально как всякое голоморфное векторное расслоение над некомпактной римановой поверхностью, см. [1; предложение 30.4]) с голоморфной связностью, монодромия которой совпадает с $\chi$ (см., например, [2; лекция 8]).

1.2. Фуксовы системы: локальная и глобальная теория. Нас будут особо интересовать так называемые фуксовы системы на сфере Римана $\overline{\mathbb{C}}$, т. е. мероморфные линейные системы (1), матрица коэффициентов которых имеет своими особенностями полюсы только первого порядка. Такая система с особыми точками $a_{1}^{0}, \ldots, a_{n}^{0}$ в комплексной плоскости имеет вид

$$
\frac{d y}{d z}=\left(\sum_{i=1}^{n} \frac{B_{i}^{0}}{z-a_{i}^{0}}\right) y, \quad y(z) \in \mathbb{C}^{p}, \quad B_{i}^{0} \in \operatorname{Mat}(p, \mathbb{C}),
$$

где матрицы $B_{i}^{0}$ удовлетворяют соотношению (2). 
Напомним некоторые факты о локальной структуре фундаментальных матриц фуксовой системы (3) в окрестности особой точки $a_{i}^{0}$. Пусть $G_{i}$ обозначает матрицу монодромии системы в точке $a_{i}^{0}$. Согласно Левелю [3], система обладает фундаментальной матрицей вида

$$
Y_{i}(z)=U_{i}(z)\left(z-a_{i}^{0}\right)^{\Lambda_{i}}\left(z-a_{i}^{0}\right)^{E_{i}},
$$

где

а) матрица $U_{i}(z)$ голоморфно обратима в точке $a_{i}^{0}$;

б) $E_{i}=\frac{1}{2 \pi \sqrt{-1}} \ln G_{i}=\operatorname{diag}\left(E_{i}^{1}, \ldots, E_{i}^{m}\right)$ - блочно-диагональная матрица, каждый блок $E_{i}^{j}$ которой является верхнетреугольной матрицей с единственным собственным значением $\rho_{i}^{j}$, и ветвь логарифма выбрана таким образом, что собственные значения матрицы $E_{i}$ удовлетворяют условию

$$
0 \leqslant \operatorname{Re} \rho_{i}^{j}<1
$$

в) $\Lambda_{i}=\operatorname{diag}\left(\Lambda_{i}^{1}, \ldots, \Lambda_{i}^{m}\right)$ - диагональная целочисленная матрица с такой же блочной структурой, как $E_{i}$, при этом диагональные элементы каждого блока $\Lambda_{i}^{j}$ образуют невозрастающую последовательность.

Матрица $Y_{i}(z)$ называется (слабо) левелевской фундаментальной матрицей. Ее столбцы образуют (слабо) левелевский базис.

Собственные значения $\beta_{i}^{j}$ матрицы-вычета $B_{i}^{0}$ называются показателями фуксовой системы $(3)$ в особой точке $a_{i}^{0}$. Используя соотношение

$$
B_{i}^{0}=\operatorname{res}_{a_{i}^{0}}\left(\frac{d Y_{i}}{d z} Y_{i}^{-1}\right),
$$

несложно проверить, что показатели совпадают с собственными значениями матрицы $\Lambda_{i}+E_{i}$. Матрицу $\Lambda_{i}$ называют матрицей нормирований фуксовой системы (3) в особой точке $a_{i}^{0}$. Согласно (5), ее диагональные элементы совпадают с целыми частями чисел $\operatorname{Re} \beta_{i}^{j}$.

Говорят, что особая точка $z=a_{i}^{0}$ фуксовой системы (3) резонанснал, если для некоторой пары $\beta_{i}^{j}, \beta_{i}^{k}$ показателей в этой точке их разность $\beta_{i}^{j}-\beta_{i}^{k}$ является ненулевым целым числом. В противном случае особенность называется нерезонансной. Из вышесказанного следует, что особая точка $a_{i}^{0}$ нерезонансна тогда и только тогда, когда все блоки $\Lambda_{i}^{j}$ матрицы $\Lambda_{i}$ из левелевского разложения (4) - скалярные. Резонансы играют важную роль при описании и параметризации фуксовых систем с помощью их локальных данных (монодромии и асимптотик, или показателей).

Две фуксовы системы с нерезонансной особой точкой $a_{i}^{0}$, имеющие одинаковую матрицу монодромии и одинаковые показатели в этой точке, локально голоморфно эквивалентны (т. е. они связаны локальным преобразованием $y^{\prime}=\Gamma(z) y$, где $\Gamma(z)$ - голоморфно обратимая в точке $a_{i}^{0}$ матрица). Действительно, левелевские фундаментальные матрицы $Y_{i}(z), Y_{i}^{\prime}(z)$ этих систем в точке $a_{i}^{0}$ имеют вид

$$
Y_{i}(z)=U_{i}(z)\left(z-a_{i}^{0}\right)^{\Lambda_{i}}\left(z-a_{i}^{0}\right)^{E_{i}}, \quad Y_{i}^{\prime}(z)=U_{i}^{\prime}(z)\left(z-a_{i}^{0}\right)^{\Lambda_{i}}\left(z-a_{i}^{0}\right)^{E_{i}^{\prime}},
$$


где матрицы $E_{i}^{\prime}=S E_{i} S^{-1}$ и $E_{i}$ сопряжены на матрицу $S=\operatorname{diag}\left(S^{1}, \ldots, S^{m}\right)$ такого же блочно-диагонального вида, как и матрицы $E_{i}, E_{i}^{\prime}$. Поскольку матрицы $\Lambda_{i}$ и $S$ коммутируют, имеем

$$
Y_{i}^{\prime}(z) S=U_{i}^{\prime}(z) S\left(z-a_{i}^{0}\right)^{\Lambda_{i}}\left(z-a_{i}^{0}\right)^{E_{i}}=U_{i}^{\prime}(z) S U_{i}^{-1}(z) Y_{i}(z)
$$

откуда следует локальная голоморфная эквивалентность двух систем.

Глобально, две фуксовы системы с одинаковыми особенностями, которые все нерезонансные, с одинаковой монодромией и показателями (во всех особых точках) связаны постоянным преобразованием $y^{\prime}=C y, C \in \mathrm{GL}(p, \mathbb{C})$. (Используя аналогичные рассуждения, можно показать, что эти системы глобально голоморфно эквивалентны, но голоморфная на всей сфере Римана матрица постоянна.) Мы не различаем такие системы, считая, таким образом, фуксову систему с $n$ фиксированными особенностями $a_{1}^{0}, \ldots, a_{n}^{0}$ элементом пространства

$$
\mathscr{M}_{a^{0}}^{*}=\left\{\left(B_{1}^{0}, \ldots, B_{n}^{0}\right) \mid B_{i}^{0} \in \operatorname{Mat}(p, \mathbb{C}), B_{1}^{0}+\cdots+B_{n}^{0}=0\right\} / \operatorname{GL}(p, \mathbb{C}) .
$$

В этом смысле может существовать только одна фуксова система с заданными нерезонансными особенностями, монодромией и показателями.

В резонансном случае появляются существенно различные фуксовы системы с одинаковыми особенностями, монодромией и показателями, образуя пространство модулей. Проиллюстрируем это примером.

ПримеР 1. Рассмотрим фуксову систему

$$
\begin{aligned}
\frac{d y}{d z}= & \left(\left(\begin{array}{cc}
1 & t_{1} \\
0 & 0
\end{array}\right) \frac{1}{z-a_{1}}+\left(\begin{array}{cc}
0 & -t_{1} \\
0 & -1
\end{array}\right) \frac{1}{z-a_{2}}\right. \\
& \left.+\left(\begin{array}{cc}
-1 & t_{2} \\
0 & 0
\end{array}\right) \frac{1}{z-a_{3}}+\left(\begin{array}{cc}
0 & -t_{2} \\
0 & 1
\end{array}\right) \frac{1}{z-a_{4}}\right) y
\end{aligned}
$$

двух уравнений с четырьмя резонансными особыми точками $a_{1}, a_{2}, a_{3}, a_{4}$, где $t_{1}, t_{2}$ - параметры. В действительности пара $\left(t_{1}, t_{2}\right)$ проективная, поскольку $\left(t_{1}, t_{2}\right)$ и $\left(\lambda t_{1}, \lambda t_{2}\right), \lambda \neq 0$, определяют одну фуксову систему в вышеозначенном смысле. В то же время, несложно проверить, что непропорциональные пары параметров задают существенно различные фуксовы системы.

Матрица коэффициентов рассматриваемой системы верхнетреугольна, поэтому система может быть легко проинтегрирована и полученная фундаментальная матрица будет иметь следующий вид:

$$
\begin{gathered}
Y(z)=\left(\begin{array}{cc}
\frac{z-a_{1}}{z-a_{3}} & 0 \\
0 & \frac{z-a_{4}}{z-a_{2}}
\end{array}\right) \\
\alpha=A t_{1}+B t_{2},
\end{gathered}
$$

где $A, B, C, D$ - некоторые рациональные функции от $a_{1}, a_{2}, a_{3}, a_{4}$. Матрица $Y(z)$ не имеет ветвления в точках $a_{3}, a_{4}$, в то время как она преобразуется в матрицы $\widetilde{Y}_{1}(z), \widetilde{Y}_{2}(z)$ после аналитических продолжений вокруг точек $a_{1}, a_{2}$ 
соответственно:

$$
\begin{aligned}
& \tilde{Y}_{1}(z)=\left(\begin{array}{cc}
\frac{z-a_{1}}{z-a_{3}} & 0 \\
0 & \frac{z-a_{4}}{z-a_{2}}
\end{array}\right)\left(\begin{array}{cc}
1 & \alpha \ln \frac{z-a_{1}}{z-a_{2}}+\frac{C t_{1}}{z-a_{1}}+\frac{D t_{1}}{z-a_{2}}+2 \pi i \alpha \\
0 & 1
\end{array}\right), \\
& \tilde{Y}_{2}(z)=\left(\begin{array}{cc}
\frac{z-a_{1}}{z-a_{3}} & 0 \\
0 & \frac{z-a_{4}}{z-a_{2}}
\end{array}\right)\left(\begin{array}{cc}
1 & \alpha \ln \frac{z-a_{1}}{z-a_{2}}+\frac{C t_{1}}{z-a_{1}}+\frac{D t_{1}}{z-a_{2}}-2 \pi i \alpha \\
0 & 1
\end{array}\right) .
\end{aligned}
$$

Таким образом, матрица $Y(z)$ имеет следующие матрицы монодромии:

$$
G_{1}=\left(\begin{array}{cc}
1 & 2 \pi i \alpha \\
0 & 1
\end{array}\right), \quad G_{2}=\left(\begin{array}{cc}
1 & -2 \pi i \alpha \\
0 & 1
\end{array}\right), \quad G_{3}=G_{4}=\mathrm{Id} .
$$

Для всех $\left(t_{1}, t_{2}\right) \in \mathbb{C}^{2}$ таких, что $\alpha=A t_{1}+B t_{2} \neq 0$, набор $\left\{G_{1}, \ldots, G_{4}\right\}$ может быть преобразован в набор

$$
G_{1}^{\prime}=\left(\begin{array}{cc}
1 & 2 \pi i \\
0 & 1
\end{array}\right), \quad G_{2}^{\prime}=\left(\begin{array}{cc}
1 & -2 \pi i \\
0 & 1
\end{array}\right), \quad G_{3}^{\prime}=G_{4}^{\prime}=\mathrm{Id}
$$

сопряжением на диагональную матрицу $\operatorname{diag}(\alpha, 1)$. Следовательно, имеется целое пространство

$$
\left\{\left(t_{1}: t_{2}\right) \in \mathbb{P}^{1}(\mathbb{C}) \mid A t_{1}+B t_{2} \neq 0\right\}
$$

фуксовых систем с одними и теми же четырьмя резонансными особенностями, одинаковыми монодромией и показателями. Оно представляет собой комплексную проективную прямую, проколотую в точке $(-B: A)$.

Есть и другой случай локальной голоморфной эквивалентности двух фуксовых систем, имеющих одинаковую матрицу монодромии $G_{i}$ и одинаковые показатели в особой точке $a_{i}^{0}$ (нерезонансной или резонансной). Это случай, когда выполнено условие

$$
\operatorname{rank}\left(G_{i}-\lambda \mathrm{Id}\right) \geqslant p-1 \quad \forall \lambda \in \mathbb{C},
$$

означающее, что каждому собственному значению матрицы $G_{i}$ соответствует единственная жорданова клетка с этим собственным значением. В таком случае жорданов базис оператора монодромии в пространстве локальных решений системы является (слабо) левелевским базисом (см. [2; пример 5.2]). Используя этот факт, можно доказать, что если все матрицы монодромии фуксовой системы удовлетворяют условию (6), то не существует другой фуксовой системы с теми же особенностями, монодромией и показателями. (Заметим, что монодромия из примера 1 не удовлетворяет условию (6), поскольку матрицы $G_{3}=G_{4}$ единичные.)

1.3. Интегрируемость пфаффовых систем. Системы, которые мы рассматриваем в данной работе, главным образом представляют собой частные

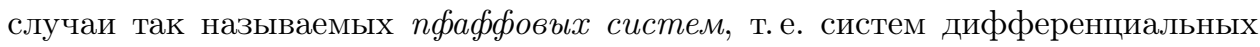
уравнений в частных производных (или обыкновенных) первого порядка вида

$$
\begin{aligned}
& d y_{1}-\left(f_{1}^{1} d z_{1}+\cdots+f_{r}^{1} d z_{r}\right)=0, \\
& \left.\ldots \ldots \ldots+\cdots \cdots \cdots+\cdots \cdots \cdots+f_{r}^{p} d z_{r}\right)=0 \\
& d y_{p}-\left(f_{1}^{p} d z_{1}+\cdots+\right.
\end{aligned}
$$


где $z_{1}, \ldots, z_{r}$ - независимые переменные, $y_{1}, \ldots, y_{p}$ - неизвестные и $f_{j}^{i}=f_{j}^{i}(z, y)$ голоморфные функции в области $D \subset \mathbb{C}^{r} \times \mathbb{C}^{p}$. Система $(7)$ называется (вполне) интегрируемой, если для любой точки $\left(z^{0}, y^{0}\right) \in D$ существует единственное решение $y(z)$ такое, что $y\left(z^{0}\right)=y^{0}$.

Интегрируемость системы (7) можно также выразить в терминах $r$-мерного голоморфного распределения $S$ на $D$, заданного данной системой. Для произвольной точки $(z, y) \in D$ рассмотрим базис $\frac{\partial}{\partial z_{1}}, \ldots, \frac{\partial}{\partial z_{r}}, \frac{\partial}{\partial y_{1}}, \ldots, \frac{\partial}{\partial y_{p}}$ касательного пространства $T_{(z, y)} D$ и $r$-мерное подпространство $S_{(z, y)} \subset T_{(z, y)} D$, порожденное векторами $\left\{\sum_{i=1}^{p} f_{j}^{i} \frac{\partial}{\partial y_{i}}+\frac{\partial}{\partial z_{j}}\right\}_{j=1}^{r}$ (таким образом, распределение $S$ порождено $r$ голоморфными векторными полями на $D$ ). Тогда интегральные многообразия системы $(7)$ - это интегральные многообразия распределения $S$. Интегрируемость системы эквивалентна при этом тому, что для любых двух локальных голоморфных векторных полей $X$ и $Y$, удовлетворяющих условию $X_{(\tilde{z}, \tilde{y})}, Y_{(\tilde{z}, \tilde{y})} \in S_{(\tilde{z}, \tilde{y})}$ для всех близких $(\tilde{z}, \tilde{y})$, то же справедливо и для их коммутатора: $[X, Y]_{(\tilde{z}, \tilde{y})} \in S_{(\tilde{z}, \tilde{y})}$.

Это можно сформулировать и на уровне кокасательного расслоения. Обозначим через $\theta_{1}, \ldots, \theta_{p}$ дифференциальные 1 -формы в левой части системы $(7)$, а через $\mathscr{I}$ - идеал дифференциальной алгебры голоморфных $k$-форм $(1 \leqslant k \leqslant$ $r+p)$ на $D$, порожденный 1 -формами $\theta_{1}, \ldots, \theta_{p}$. Тогда имеет место условие интегрируемости Фробениуса пфаффовой системы (7) (или распределения $S$ ).

Теорема 1. Система (7) интегрируема тогда и только тогда, когда

$$
d \theta_{1}, \ldots, d \theta_{p} \in \mathscr{I} .
$$

Доказательство можно найти в [4; теорема 5.1, с. 13] (в частном случае $r=2$, $p=1$ ) или в $[5]^{1}$. Условие интегрируемости Фробениуса можно представлять себе как обобщение теоремы Коши для обыкновенных дифференциальных уравнений на случай уравнений в частных производных. Нетрудно проверить, что условие интегрируемости всегда выполняется при $r=1$.

ПримеР 2. Рассмотрим алгебру рациональных функций от переменных $t=$ $\left(t_{1}, \ldots, t_{r}\right) \in \mathbb{C}^{r},(p, q)=\left(p_{1}, \ldots, p_{n}, q_{1}, \ldots, q_{n}\right) \in \mathbb{C}^{2 n}$ и скобку Пуассона $\{\cdot, \cdot\}$ на ней, которая паре функций $f, g$ ставит в соответствие функцию

$$
\{f, g\}=\sum_{i=1}^{n}\left(\frac{\partial f}{\partial q_{i}} \frac{\partial g}{\partial p_{i}}-\frac{\partial f}{\partial p_{i}} \frac{\partial g}{\partial q_{i}}\right) .
$$

Тогда пфаффова система

$$
\begin{array}{rlll}
d p_{1} & =\sum_{j=1}^{r}\left\{H_{j}, p_{1}\right\} d t_{j}, \quad \ldots, \quad d p_{n}=\sum_{j=1}^{r}\left\{H_{j}, p_{n}\right\} d t_{j}, \\
d q_{1} & =\sum_{j=1}^{r}\left\{H_{j}, q_{1}\right\} d t_{j}, \quad \ldots, \quad d q_{n}=\sum_{j=1}^{r}\left\{H_{j}, q_{n}\right\} d t_{j}
\end{array}
$$

${ }^{1} \mathrm{~B}[5]$ рассматриваются гладкие, а не голоморфные распределения, но доказательство применимо и к последним. 
называется гамильтоновой системой с гамильтонианами $H_{j}=H_{j}(t, p, q)$. Такая система также может быть записана в виде

$$
\frac{\partial p_{i}}{\partial t_{j}}=\frac{\partial H_{j}}{\partial q_{i}}, \quad \frac{\partial q_{i}}{\partial t_{j}}=-\frac{\partial H_{j}}{\partial p_{i}}, \quad i=1, \ldots, n, \quad j=1, \ldots, r .
$$

Условие интегрируемости для гамильтоновой системы (8) формулируется следующим образом (см. [4; предложение 6.4, с. 23]). Положим

$$
\Gamma_{i j}=\frac{\partial H_{j}}{\partial t_{i}}-\frac{\partial H_{i}}{\partial t_{j}}+\left\{H_{i}, H_{j}\right\}
$$

Тогда система (8) интегрируема в том и только том случае, когда

$$
\left\{\Gamma_{j k}, p_{i}\right\}=\left\{\Gamma_{j k}, q_{i}\right\}=0, \quad i=1, \ldots, n, \quad j, k=1, \ldots, r,
$$

т. е. все $\Gamma_{j k}$ не зависят от $(p, q)$.

Важным частным случаем пфаффовых систем является линейная система (записанная в матричном виде)

$$
d y=\Omega y, \quad y(z) \in \mathbb{C}^{p},
$$

где $\Omega$ - голоморфная матричная дифференциальная 1-форма на $U \subset \mathbb{C}^{r}$. Например, фуксова система (3) представляет собой систему вида (9), где $r=1$ и форма $\Omega$ мероморфна на $\overline{\mathbb{C}}$, с полюсами $a_{1}^{0}, \ldots, a_{n}^{0}$ первого порядка.

Очевидно, что множество локальных решений системы (9) в окрестности любой точки $z^{0} \in U$ образует векторное пространство, и интегрируемость системы эквивалентна тому, что эти пространства $p$-мерны. С другой стороны, условие интегрируемости Фробениуса для системы (9) преобразуется в следующее утверждение.

СлЕДСтвие 1. Система (9) интегрируема тогда и только тогда, когда

$$
d \Omega=\Omega \wedge \Omega .
$$

ДокАЗАтЕльство. Если система (9) интегрируема, то существует локальная голоморфная $(p \times p)$-матрица $Y(z)$ такая, что $\Omega=d Y \cdot Y^{-1}$. Поэтому ${ }^{2}$

$$
d \Omega=-d Y \wedge d\left(Y^{-1}\right)=d Y \wedge Y^{-1} \cdot d Y \cdot Y^{-1}=d Y \cdot Y^{-1} \wedge d Y \cdot Y^{-1}=\Omega \wedge \Omega .
$$

Обратно, если выполнено соотношение (10), то дифференциальные 1-формы $\theta_{i}=d y_{i}-\sum_{j=1}^{p} \Omega_{i j} y_{j}$ удовлетворяют условию теоремы 1 и система (9) интегрируема. Действительно,

$$
\begin{aligned}
d \theta_{i} & =-\sum_{j=1}^{p}\left(d \Omega_{i j}\right) y_{j}+\sum_{j=1}^{p} \Omega_{i j} \wedge d y_{j}=-\sum_{j=1}^{p}\left(d \Omega_{i j}\right) y_{j}+\sum_{j=1}^{p} \Omega_{i j} \wedge\left(\theta_{j}+\sum_{k=1}^{p} \Omega_{j k} y_{k}\right) \\
& =\sum_{k=1}^{p}\left(-d \Omega_{i k}+\sum_{j=1}^{p} \Omega_{i j} \wedge \Omega_{j k}\right) y_{k}+\sum_{j=1}^{p} \Omega_{i j} \wedge \theta_{j}=\sum_{j=1}^{p} \Omega_{i j} \wedge \theta_{j} .
\end{aligned}
$$

Следствие доказано.

\footnotetext{
${ }^{2}$ Следует помнить о том, что для функции $f$ и дифференциальной 1-формы $\sigma$ имеет место соотношение $d(f \sigma)=f d \sigma+d f \wedge \sigma$, в то время как $d(\sigma f)$ (что то же самое) представляется в виде $d(\sigma f)=(d \sigma) f-\sigma \wedge d f$.
} 
Линейная пфаффова система (9) снова допускает описание в терминах голоморфных векторных расслоений и связностей. Рассмотрим тривиальное векторное расслоение $E=U \times \mathbb{C}^{p}$ ранга $p$ над $U$ и аналогичный базис $\left\{s_{i}\right\}$ сечений, соответствующий стандартному базису $\left\{e_{i}\right\}$ пространства $\mathbb{C}^{p}$. Матрица

$$
\Omega=\sum_{i=1}^{r} C_{i} d z_{i}
$$

голоморфных дифференциальных 1-форм на $U$ определяет в базисе $\left\{s_{i}\right\}$ голоморфную связность $\nabla$ в расслоении $E$ :

$$
\nabla:\left(s_{1}, \ldots, s_{p}\right) \mapsto-\left(s_{1}, \ldots, s_{p}\right) \Omega .
$$

Связность $\nabla: \Gamma^{0}(E) \rightarrow \Gamma^{1}(E)$ допускает естественное продолжение $\widetilde{\nabla}: \Gamma^{1}(E) \rightarrow$ $\Gamma^{2}(E)$ из пространства $\Gamma^{0}(E)$ голоморфных сечений расслоения $E$ в пространство $\Gamma^{1}(E)$ голоморфных дифференциальных 1-форм со значениями в $E$ по формуле

$$
\widetilde{\nabla}(s \sigma)=s d \sigma+\nabla(s) \wedge \sigma,
$$

где $s \in \Gamma^{0}(E)$ и $\sigma$ - голоморфная дифференциальная 1-форма на $U$. Пространство горизонтальных сечений (тех $y=\sum_{i=1}^{p} y_{i} s_{i}$, для которых $\left.\nabla(y)=0\right) p$-мерно тогда и только тогда, когда кривизна $\widetilde{\nabla} \circ \nabla: \Gamma^{0}(E) \rightarrow \Gamma^{2}(E)$ связности $\nabla$ нулевая, т. е. 2-форма кривизны (в базисе $\left.\left\{s_{i}\right\}\right)$ есть

$$
-d \Omega+(-\Omega) \wedge(-\Omega)=0
$$

что эквивалентно интегрируемости пфаффовой системы (9). Связность $\nabla$ тогда также называется интегрируемой (или плоской). В терминах матриц $C_{i}$ условие интегрируемости связности $\nabla$ переписывается в виде так называемых уравнений нулевой кривизны

$$
\frac{\partial C_{j}}{\partial z_{i}}-\frac{\partial C_{i}}{\partial z_{j}}-\left[C_{i}, C_{j}\right]=0, \quad i, j=1, \ldots, r .
$$

Вышеприведенное описание соответствующим образом переносится на нетривиальные расслоения. Так, для (нетривиального) голоморфного векторного расслоения $E$ ранга $p$ над комплексным многообразием $Z$ можно ввести такие понятия, как (голоморфная) связность, интегрируемая связность, горизонтальные сечения и монодромия интегрируемой связности. Для определения последней необходимо, чтобы пространство локальных горизонтальных сечений связности было $p$-мерным, что обеспечивается интегрируемостью связности.

\section{2. Интегрируемые деформации мероморфных связностей}

Для пространства $U=\overline{\mathbb{C}} \backslash\left\{a_{1}^{0}, \ldots, a_{n}^{0}\right\}$ выше (см. конец п. 1.1) была установлена связь между интегрируемой голоморфной связностью $\nabla^{0}$ в (тривиальном) голоморфном векторном расслоении ранга $p$ над $U$ и $p$-мерным представлени- 
ем фундаментальной группы $\pi_{1}(U)$. Это соответствие имеет место и в общем случае.

Пусть $E$ - голоморфное векторное расслоение ранга $p$ над комплексным многообразием $X$ и $\nabla$ - интегрируемая связность в $E$. Имеется важное соответствие между такими парами $(E, \nabla)$ и $\mathrm{GL}(p, \mathbb{C})$-представлениями фундаментальной группы пространства $X$, которое мы будем широко использовать в дальнейшем. А именно, справедливо следующее утверждение (см. [6; предложение 2.5]).

ТеОРема 2. Если $X$ связно, то имеет место эквивалентность между категорией пар $(E, \nabla)$, состоящих из голоморфного векторного расслоения $E$ ранга р над $X$ и интегрируемой связности $\nabla$, и категорией р-мерных представлений группы $\pi_{1}(X)$. Это соответствие задается естественным действием группы $\pi_{1}(X)$ на слой расслоения $E$ над некоторой базисной точкой пространства $X$.

Связности, определяемые системами вида (1), заданы не на всей сфере Римана $\overline{\mathbb{C}}$ - они обладают особенностями ввиду мероморфности матрицы $B(z)$ на $\overline{\mathbb{C}}$. Поэтому можно сказать, что система (1) определяет мероморфную связность $\nabla^{0}$ в тривиальном векторном расслоении $E^{0}=\overline{\mathbb{C}} \times \mathbb{C}^{p}$ ранга $p$ над $\overline{\mathbb{C}}-$ линейное отображение из пространства $M^{0}\left(E^{0}\right)$ мероморфных сечений расслоения $E^{0}$ в пространство $M^{1}\left(E^{0}\right)$ мероморфных дифференциальных 1-форм на $\overline{\mathbb{C}}$ со значениями в $E^{0}$. Теперь представим данное понятие мероморфной связности в общей ситуации. Пусть $X$ - комплексное многообразие размерности $m$, $Y \subset X$ - аналитическое подмножество коразмерности один и $E$ - голоморфное векторное расслоение ранга $p$ над $X$. Если $X$ связно, то пространство $X \backslash Y$ также связно (см. [7; теорема 16.2]). Допустим, в ограничении $\left.E\right|_{X \backslash Y}$ введена интегрируемая связность $\nabla$.

ОПРЕДЕЛЕНИЕ 1. Связность $\nabla$ называется мероморфной над $Y$, если для любой точки $y \in Y$ найдется ее окрестность $U$ такая, что расслоение $\left.E\right|_{U}$ тривиально и форма связности $\nabla$ в базисе его голоморфных сечений мероморфна в $U$, имея $U \cap Y$ своим полярным множеством.

Заметим, что данное понятие не зависит от выбора базиса локальных голоморфных сечений. Напомним, что функция $f$ мероморфна в $U$, если она голоморфна в $U \backslash P$, не может быть продолжена голоморфно в $P$ и представляется в виде отношения $f(t)=\varphi(t) / \psi(t)$ голоморфных функций в окрестности каждой точки $t^{*} \in P$ (следовательно, $\left.\psi\left(t^{*}\right)=0\right)$, где $P \subset U$ - аналитическое подмножество коразмерности один (определяемое локально уравнением $\psi(t)=0)$. Множество $P$ называется полярным множеством мероморфной функции $f$. Точки этого множества делятся на полюсы (где функция $\varphi$ не обращается в нуль) и точки неопределенности (в которых $\varphi=0)$.

Связность, определяемая фуксовой системой (3), служит примером следующего класса связностей $\nabla$, мероморфных над $Y$.

ОПРЕДЕЛЕНИЕ 2. Говорят, что связность $\nabla$, мероморфная над $Y$, имеет логарифмический полюс над $Y$, если для любой регулярной точки $y \in Y$ найдется ее окрестность $U$ с координатами $\left(t_{1}, \ldots, t_{m}\right)$ такая, что множество $Y \cap U$ задается уравнением $\left\{t_{1}=0\right\}$ и форма $\Omega_{U}$ связности $\nabla$ в базисе голоморфных 
сечений расслоения $\left.E\right|_{U}$ имеет вид

$$
\Omega_{U}=\frac{A_{1}}{t_{1}} d t_{1}+\sum_{i=2}^{m} A_{i} d t_{i}
$$

где все $(p \times p)$-матрицы $A_{i}$ голоморфны в $U$.

Мы будем кратко называть $\nabla$ мероморфной (соответственно логарифмической) связностью в голоморфном векторном расслоении $E$. Множество $Y$ называется полярным множеством связности $\nabla$.

Начальной точкой для интегрируемых деформаций является мероморфная линейная система на $\overline{\mathbb{C}}$, имеющая особенности $a_{1}^{0}, \ldots, a_{n}^{0} \in \mathbb{C}$. В более геометрических терминах это:

- голоморфно тривиальное векторное расслоение $E^{0}$ ранга $p$ над $\overline{\mathbb{C}}$,

- интегрируемая мероморфная связность $\nabla^{0}$ в расслоении $E^{0}$, с особенностями $a_{1}^{0}, \ldots, a_{n}^{0}$.

Поскольку на $\overline{\mathbb{C}}$ нет ненулевых голоморфных дифференциальных 1-форм, то форма $\omega^{0}$ связности $\nabla^{0}$ может быть записана в виде

$$
\omega^{0}=\sum_{i=1}^{n} \sum_{l \geqslant 1} \frac{B_{i l}^{0}}{\left(z-a_{i}^{0}\right)^{l}} d z, \quad \sum_{i=1}^{n} B_{i 1}^{0}=0 .
$$

Интересующие нас деформации заключаются в перемещении полюсов $\left\{a_{i}^{0}\right\}$ формы $\omega^{0}$ внутри $\mathbb{C}$. Точнее, способ перемещения полюсов определяется связным комплексным многообразием $T$, кратко именуемым пространством деформации, и набором так называемых функций деформации - голоморфных функций

$$
a_{i}: T \rightarrow \mathbb{C}, \quad i=1, \ldots, n .
$$

При этом должна существовать начальная точка $t^{0} \in T$ такая, что

$$
a_{i}\left(t^{0}\right)=a_{i}^{0}, \quad i=1, \ldots, n .
$$

Мы ограничимся деформациями, при которых полюсы не сливаются, т. е. $a_{i}(t) \neq a_{j}(t)$ для всех $t \in T$ и $i \neq j$. Приведем некоторые примеры пространств деформации, которые будут использоваться в дальнейшем.

Пример 3. 1) $T=D\left(a^{0}\right)$ - диск малого радиуса с центром в точке $a^{0}=$ $\left(a_{1}^{0}, \ldots, a_{n}^{0}\right)$ пространства

$$
Z=\mathbb{C}^{n} \backslash \bigcup_{i \neq j}\left\{a_{i}=a_{j}\right\}
$$

В данном случае параметр $a=\left(a_{1}, \ldots, a_{n}\right) \in D\left(a^{0}\right)$ деформации - набор полюсов, а функции деформации - проекции на все координаты, так что мы пишем просто $a_{i}$ вместо $a_{i}(a)$.

2) $T=D\left(a^{0}\right) \times \mathscr{W}$, где $\mathscr{W}-$ связное комплексное многообразие. В этом случае мы для удобства разделяем переменные $a \in D\left(a^{0}\right)$ и $w \in \mathscr{W}$. Функция деформации с номером $i$ есть $(a, w) \mapsto a_{i}$, и мы снова пишем $a_{i}$ вместо $a_{i}(a, w)$. 
3) $T=\widetilde{Z}$ - универсальное накрытие пространства $Z$. Тогда $i$-я функция деформации есть композиция накрытия $\widetilde{Z} \rightarrow Z$ и проекции на $i$-ю координату. Поскольку $\widetilde{Z}$ является универсальным накрывающим пространством, его фундаментальная группа тривиальна. Используя точную последовательность гомотопических групп, происходящую из локально тривиального расслоения

$$
\mathbb{C}^{n} \backslash \bigcup_{i \neq j}\left\{a_{i}=a_{j}\right\} \rightarrow \mathbb{C}^{n-1} \backslash \bigcup_{i \neq j}\left\{a_{i}=a_{j}\right\},
$$

определяемого проекцией вдоль последней координаты, и индукцию по $n$, можно проверить, что все гомотопические группы $\pi_{k}(\widetilde{Z})=\pi_{k}(Z), k \geqslant 2$, также тривиальны (см., например, [2; дополнение 4]). Тогда из теоремы Уайтхеда (см. [8; § 11.5]) следует, что пространство $\widetilde{Z}$ стягиваемо.

Пространство $\widetilde{Z}$ обладает еще одним важным свойством, а именно, оно является многообразием Штейна как универсальное накрывающее пространство многообразия Штейна $Z$ (согласно [9] всякое неразветвленное накрытие многообразия Штейна само является многообразием Штейна). А поскольку пространство $\widetilde{Z}$ стягиваемо, то к нему применима теорема Грауэрта и, следовательно, любое голоморфное векторное расслоение над $\widetilde{Z}$ голоморфно тривиально (см. [10]).

Обозначим теперь через $X$ многообразие $\overline{\mathbb{C}} \times T$. Внутри $X$ рассмотрим подмногообразие $Y=Y_{1} \cup \cdots \cup Y_{n}$, заданное соотношениями

$$
Y_{i}=\left\{(z, t) \in X \mid z-a_{i}(t)=0\right\}, \quad i=1, \ldots, n .
$$

Итак, мы имеем все для того, чтобы ввести следующее понятие.

ОПредЕЛЕНИЕ 3. Интегрируемая деформация $(E, \nabla)$ пары $\left(E^{0}, \nabla^{0}\right)$ с пространством деформации $T$, функциями деформации $\left\{a_{i}(t)\right\}$ и начальной точкой $t^{0} \in T$ состоит из

- голоморфного векторного расслоения $E$ ранга $p$ над $X=\overline{\mathbb{C}} \times T$,

- интегрируемой мероморфной связности $\nabla$ в $E$, полярное множество которой есть $Y$, такой, что ограничение пары $(E, \nabla)$ на $\overline{\mathbb{C}} \times\left\{t^{0}\right\}$ изоморфно $\left(E^{0}, \nabla^{0}\right)$.

Задавшись пространством деформации $T$, функциями деформации $\left\{a_{i}(t)\right\}$ и начальной точкой $t^{0} \in T$, уместно спросить о существовании интегрируемой деформации с этими данными. Здесь можно столкнуться с топологическими препятствиями.

Пусть $i: \overline{\mathbb{C}} \hookrightarrow \overline{\mathbb{C}} \times T-$ вложение, $i(z)=\left(z, t^{0}\right)$. Оно индуцирует естественное отображение

$$
i_{*}: \pi_{1}\left(\overline{\mathbb{C}} \backslash\left\{a_{1}^{0}, \ldots, a_{n}^{0}\right\}\right) \rightarrow \pi_{1}(X \backslash Y) .
$$

В силу теоремы 2 из существования интегрируемой деформации $(E, \nabla)$ пары $\left(E^{0}, \nabla^{0}\right)$ следует, что представление $\chi$ группы $\pi_{1}\left(\overline{\mathbb{C}} \backslash\left\{a_{1}^{0}, \ldots, a_{n}^{0}\right\}\right)$, соответствующее паре $\left(E^{0}, \nabla^{0}\right)$, изоморфно $\widetilde{\chi} \circ i_{*}$, где $\tilde{\chi}$ - представление группы $\pi_{1}(X \backslash Y)$, соответствующее паре $(E, \nabla)$. Рассмотрим локально тривиальное расслоение

$$
p_{2}: X \backslash Y \rightarrow T, \quad p_{2}(z, t)=t,
$$


над $T$ со слоем $p_{2}^{-1}\left(t^{0}\right)=\overline{\mathbb{C}} \backslash\left\{a_{1}^{0}, \ldots, a_{n}^{0}\right\}$. Соответствующая длинная точная последовательность

$$
\cdots \longrightarrow \pi_{2}(T) \stackrel{\delta}{\longrightarrow} \pi_{1}\left(\overline{\mathbb{C}} \backslash\left\{a_{1}^{0}, \ldots, a_{n}^{0}\right\}\right) \stackrel{i_{*}}{\longrightarrow} \pi_{1}(X \backslash Y) \longrightarrow \pi_{1}(T)
$$

гомотопических групп влечет то, что представление $\chi$, продолжающееся до $\widetilde{\chi}$, еще и должно быть тривиальным на образе отображения $\delta$, поскольку $\chi \circ \delta=$ $\widetilde{\chi} \circ\left(i_{*} \circ \delta\right) \equiv 1$. Эти вопросы решаются сами собой, если $\pi_{1}(T)=\pi_{2}(T)=\{1\}$. Таким образом, для пространств $T=D\left(a^{0}\right)$ или $T=\widetilde{Z}$ из примера 3 и соответствующих функций деформации нет топологических препятствий к существованию интегрируемой деформации с этими данными, что оказывается достаточным для того, чтобы следующее утверждение имело место.

Теорема 3. Для любой парь $\left(E^{0}, \nabla^{0}\right)$ существует ее интегрируемая деформачия $(E, \nabla)$ с пространством деформации $\widetilde{Z}$ и соответствующими функииями деформации $\left\{a_{i}(t)\right\}$.

ДоказАтельство. Сначала построим пару $(E, \nabla)$ над $X \backslash Y$. Из точной последовательности (11) гомотопических групп мы заключаем, что отображение $i_{*}$ является изоморфизмом. Поэтому $p$-мерное представление группы $\pi_{1}\left(\overline{\mathbb{C}} \backslash\left\{a_{1}^{0}, \ldots, a_{n}^{0}\right\}\right)$, соответствующее паре $\left(\left.E^{0}\right|_{\overline{\mathbb{C}} \backslash\left\{a_{1}^{0}, \ldots, a_{n}^{0}\right\}}, \nabla^{0}\right)$, определяет $p$-мерное представление группы $\pi_{1}(X \backslash Y)$, и, таким образом, мы получаем пару $(E, \nabla)$ над $X \backslash Y$. Остается продолжить векторное расслоение $E$ на все многообразие $X$ и показать, что связность $\nabla$ мероморфна над $Y$. Проделаем это на непересекающихся окрестностях компонент подмногообразия $Y$. Введем некоторые обозначения.

Для каждой точки $t \in \widetilde{Z}$ обозначим через $\delta(t)$ минимум расстояний $\left\{\left|a_{i}(t)-a_{j}(t)\right|,\left|a_{i}^{0}-a_{j}^{0}\right|\right\}_{i \neq j}$ и через $D_{j}(t)-$ диск

$$
D_{j}(t)=\left\{z \in \mathbb{C}|| z-a_{j}(t) \mid<\frac{\delta(t)}{4}\right\}
$$

радиуса $\delta(t) / 4$ с центром в точке $a_{j}(t) \in \mathbb{C}$. Далее, определим окрестность $V_{j}$ компоненты $Y_{j}$ как

$$
V_{j}=\left\{(z, t) \in X \mid z \in D_{j}(t)\right\}, \quad j=1, \ldots, n .
$$

По построению эти открытые окрестности не пересекаются. Проекция $(z, t) \mapsto t$ отображает множества $V_{j} \backslash Y_{j}$ на $\widetilde{Z}$ так, что слой над точкой $t^{0} \in \widetilde{Z}$ есть $D_{j}\left(t^{0}\right) \backslash\left\{a_{j}^{0}\right\}$. Следовательно, эти множества суть локально тривиальные расслоения с базой $\widetilde{Z}$ и слоем $D_{j}\left(t^{0}\right) \backslash\left\{a_{j}^{0}\right\}$. Тогда из соответствующей длинной точной последовательности

$$
\cdots \longrightarrow \pi_{2}(\widetilde{Z}) \longrightarrow \pi_{1}\left(D_{j}\left(t^{0}\right) \backslash\left\{a_{j}^{0}\right\}\right) \stackrel{i_{*}}{\longrightarrow} \pi_{1}\left(V_{j} \backslash Y_{j}\right) \longrightarrow \pi_{1}(\widetilde{Z})
$$

гомотопических групп следует, что отображение $i_{*}$ индуцирует изоморфизм групп $\pi_{1}\left(D_{j}\left(t^{0}\right) \backslash\left\{a_{j}^{0}\right\}\right)$ и $\pi_{1}\left(V_{j} \backslash Y_{j}\right)$.

Рассмотрим сюръективные отображения

$$
p_{j}: V_{j} \rightarrow D_{j}\left(t^{0}\right), \quad p_{j}(z, t)=z-a_{j}(t)+a_{j}^{0}, \quad j=1, \ldots, n,
$$


и векторные расслоения $E_{j}$ над $V_{j}$ - обратные прообразы расслоения $\left.E^{0}\right|_{D_{j}\left(t^{0}\right)}$ под действием этих отображений. Пусть $\omega^{0}$ обозначает форму связности $\nabla^{0}$ в базисе $\left\{s_{1}, \ldots, s_{p}\right\}$ голоморфных сечений последнего расслоения. Тогда связность $\nabla_{j}$ в расслоении $\left.E_{j}\right|_{V_{j} \backslash Y_{j}}$ задается формой $\Omega_{j}$, равной $p_{j}^{*}\left(\omega^{0}\right)$ в базисе $\left\{p_{j}^{*}\left(s_{1}\right), \ldots, p_{j}^{*}\left(s_{p}\right)\right\}$. В частности, форму $\Omega_{j}$ в произвольном базисе голоморфных сечений расслоения $E_{j}$ можно представить в виде

$$
\Omega_{j}=\sum_{l \geqslant 1} \frac{B_{j l}(t)}{\left(z-a_{j}(t)\right)^{l}} d\left(z-a_{j}(t)\right)+w_{j}
$$

где $w_{j}$ - голоморфная матричная дифференциальная 1-форма на $V_{j}$ и $\left\{B_{j l}\right\}$ голоморфные $(p \times p)$-матрицы на $\widetilde{Z}$, удовлетворяющие условию $B_{j l}=0$ при всех достаточно больших $l$. Поскольку

$$
d p_{j}^{*}\left(\omega^{0}\right)=p_{j}^{*}\left(d \omega^{0}\right)=p_{j}^{*}\left(\omega^{0} \wedge \omega^{0}\right)=p_{j}^{*}\left(\omega^{0}\right) \wedge p_{j}^{*}\left(\omega^{0}\right)
$$

то форма $\Omega_{j}=p_{j}^{*}\left(\omega^{0}\right)$ удовлетворяет условию (10) и связность $\nabla_{j}$ интегрируема. Далее, связность $\nabla_{j}$ мероморфна над $Y_{j}$, так как мероморфна форма $\Omega_{j}$, и обратный прообраз ограничения пары $\left(E_{j}, \nabla_{j}\right)$ на $D_{j}\left(t^{0}\right) \times\left\{t^{0}\right\}$ под действием отображения $i$ изоморфен паре $\left(\left.E^{0}\right|_{D_{j}\left(t^{0}\right)}, \nabla^{0}\right)$. Как видно, представления монодромии пар $\left(\left.E\right|_{V_{j} \backslash Y_{j}}, \nabla\right)$ и $\left(\left.E_{j}\right|_{V_{j} \backslash Y_{j}}, \nabla_{j}\right)$ совпадают (будучи равными монодромии пары $\left.\left(\left.E^{0}\right|_{D_{j}}\left(t^{0}\right), \nabla^{0}\right)\right)$. Поэтому эти пары изоморфны, и так мы получаем продолжение пары $(E, \nabla)$ на $X$. Это завершает доказательство теоремы ${ }^{3}$.

ЗАмЕчАниЕ 1. Из доказательства теоремы следует, что если исходная связность $\nabla^{0}$ логарифмическая, то логарифмической будет и связность $\nabla$.

ЗАмЕчАнИЕ 2. Ясно, что конструкция теоремы 3 применима и в случае, если заменить пространство $\widetilde{Z}$ произвольным пространством деформации $T$, для которого $\pi_{1}(T)=\pi_{2}(T)=\{1\}$, например, диском $D\left(a^{0}\right)$ малого радиуса с центром в точке $a^{0}$. Интегрируемые деформации также существуют для пространства деформации $T=D\left(a^{0}\right) \times \mathscr{W}$, где $\mathscr{W}-$ связное комплексное многообразие. В этом случае можно взять обратный прообраз интегрируемой деформации на $\overline{\mathbb{C}} \times D\left(a^{0}\right)$ под действием стандартной проекции $\overline{\mathbb{C}} \times T \rightarrow \overline{\mathbb{C}} \times D\left(a^{0}\right)$.

Если пространство деформации $T$ односвязно, то функции деформации $a_{i}(t)$ задают голоморфное отображение

$$
a: T \rightarrow Z, \quad a(t)=\left(a_{1}(t), \ldots, a_{n}(t)\right),
$$

которое поднимается до отображения $\tilde{a}: T \rightarrow \widetilde{Z}$. Таким образом, имеется голоморфное отображение

$$
f: \overline{\mathbb{C}} \times T \rightarrow \overline{\mathbb{C}} \times \widetilde{Z}, \quad(z, t) \mapsto(z, \tilde{a}(t)) .
$$

\footnotetext{
ЗЗаметим, что каждое отображение $p_{j}$ переводит соответствующую гиперповерхность $Y_{j}$ в особую точку $a_{j}^{0}$ связности $\nabla^{0}$, и поэтому $Y=\bigcup_{i=1}^{n} Y_{i}$ есть полярное множество построенной связности $\nabla$. Если же вместо локальных отображений использовать простую глобальную конструкцию с проекцией $\overline{\mathbb{C}} \times \widetilde{Z} \rightarrow \overline{\mathbb{C}}$ и обратным прообразом пары $\left(E^{0}, \nabla^{0}\right)$ под действием этой проекции, то мы не получим нужное полярное множество.
} 
Тогда обратный прообраз $\left(f^{*}(E), f^{*}(\nabla)\right)$ интегрируемой деформации $(E, \nabla)$, полученной в теореме 3 , также будет интегрируемой деформацией (множество $\bigcup_{i=1}^{n}\left\{z-a_{i}(t)=0\right\}$ переходит в полярное множество связности $\nabla$ под действием отображения $f$ ).

ЗАмечАнИЕ 3. Пусть $(E, \nabla)$ - интегрируемая деформация, построенная выше. Тогда она определяет интегрируемую связность $\nabla(\infty)$ в ограничении $\left.E\right|_{\{\infty\} \times T}$.

Заметим, что ограничения интегрируемой деформации $(E, \nabla)$ на $\overline{\mathbb{C}} \times\{t\}$, $t \in T$, определяют семейство $\left(E_{t}, \nabla_{t}\right)_{t \in T}$ голоморфных векторных расслоений ранга $p$ над $\overline{\mathbb{C}}$ с мероморфными связностями. Если пространство деформации $T$ односвязно, то монодромия связности $\nabla_{t}$ продолжается голоморфно на $T$ как функция параметра $t$. При доказательстве теоремы 3 было показано, что в случае тривиальности группы $\pi_{2}(T)$ эта функция постоянна, т. е. деформация сохраняет монодромию связности $\nabla_{t}$ (последняя совпадает с монодромией связности $\nabla$ при всех $t \in T)$.

Далее мы рассмотрим две основные цели данной работы:

1) Пусть $(E, \nabla)$ - интегрируемая деформация, полученная в теореме 3 (и замечании 2). Первая цель - описание множества тех $t \in T$, для которых расслоение $E_{t}$ голоморфно тривиально.

2) Пусть $(E, \nabla)$ - интегрируемая деформация логарифмической связности $\nabla^{0}$ такая, что расслоение $E$ голоморфно тривиально для некоторого пространства деформации (это случай изомонодромных деформаций фуксовых систем, который будет рассмотрен в следующем разделе). Вторая цель - описать форму $\Omega$ связности $\nabla$.

Для простоты мы не будем рассматривать в данной статье изомонодромные деформации систем (1) с иррегулярными (нефуксовыми) особенностями. Традиционно от таких деформаций требуется сохранение не только монодромии, но и матриц Стокса исходной системы. Эти деформации представлены и частично изучены в работах [11]--[14].

\section{3. Изомонодромные деформации фуксовых систем}

3.1. Общая теория. Изомонодромные деформации фуксовых систем можно определить следующим образом. Рассмотрим семейство

$$
\frac{d y}{d z}=\left(\sum_{i=1}^{n} \frac{B_{i}(t)}{z-a_{i}(t)}\right) y, \quad y(z) \in \mathbb{C}^{p}, \quad B_{i}(t) \in \operatorname{Mat}(p, \mathbb{C}),
$$

фуксовых систем, голоморфно зависящее от параметра $t \in T$, где $T$ - односвязное комплексное многообразие с базисной точкой $t^{0}$. Семейство (13) определено на пространстве

$$
(\overline{\mathbb{C}} \times T) \backslash \bigcup_{i=1}^{n}\left\{z-a_{i}(t)=0\right\}
$$


Мы предполагаем, что особенности семейства (13) не сливаются, т. е. $a_{i}(t) \neq$ $a_{j}(t)$ для всех $t \in T$ и $i \neq j$.

ОПРЕДЕЛЕНИЕ 4. Говорят, что семейство (13) изомонодромно или, более детально, является изомонодромной деформацией фуксовой системы (3), если $a_{i}\left(t^{0}\right)=a_{i}^{0}, B_{i}\left(t^{0}\right)=B_{i}^{0}$ и для всех точек $t \in T$ представления монодромии

$$
\chi: \pi_{1}\left(\overline{\mathbb{C}} \backslash\left\{a_{1}(t), \ldots, a_{n}(t)\right\}\right) \rightarrow \operatorname{GL}(p, \mathbb{C})
$$

соответствующих систем одинаковы. Это означает, что для каждой точки $t \in T$ найдется фундаментальная матрица $Y(z, t)$ соответствующей системы (13) такая, что ее монодромия не зависит от $t$. Матрица $Y(z, t)$ называется изомонодромной фундаментальной матрищей.

Сделаем некоторые пояснения к этому определению. Пусть $Y^{0}(z, t)$ - фундаментальная матрица семейства (13), удовлетворяющая начальному условию $Y^{0}\left(z_{0}, t\right)=\mathrm{Id}$ и, таким образом, аналитически зависящая от $t$ ввиду соответствующей теоремы. Поскольку пространство $T$ односвязно, матрицы монодромии $G_{1}(t), \ldots, G_{n}(t)$, соответствующие матрице $Y^{0}(z, t)$, суть голоморфные функции на всем пространстве $T$. Тогда семейство (13) изомонодромно, если для каждой точки $t \in T$ найдется матрица $C(t) \in \mathrm{GL}(p, \mathbb{C})$ такая, что

$$
C^{-1}(t) G_{i}(t) C(t)=\text { const. }
$$

Другими словами, матрицы монодромии, соответствующие фундаментальной матрице $Y(z, t)=Y^{0}(z, t) C(t)$, не зависят от $t$. Если пространство $T$ является стягиваемым многообразием Штейна, то согласно результату А. А. Болибруха (подробнее см. [15] или [2; теорема 13.1]) матричная функция $C(t)$ может быть выбрана голоморфной ${ }^{4}$. Следовательно, изомонодромная фундаментальная матрица $Y(z, t)$ также голоморфно зависит от $t$.

Фуксова система (3) определяет логарифмическую связность $\nabla^{0}$ в голоморфно тривиальном векторном расслоении $E^{0}$ ранга $p$ над $\overline{\mathbb{C}}$. В силу теоремы 3 и замечаний 1,2 существует интегрируемая деформация $(E, \nabla)$ пары $\left(E^{0}, \nabla^{0}\right)$ с пространством деформации $T$ и функциями деформации $\left\{a_{i}(t)\right\}$, при этом связность $\nabla$ логарифмическая. Как упоминалось выше (см. конец раздела 2$)$, ограничения пары $(E, \nabla)$ на $\overline{\mathbb{C}} \times\{t\}, t \in T$, определяют семейство $\left(E_{t}, \nabla_{t}\right)_{t \in T}$ голоморфных векторных расслоений над $\overline{\mathbb{C}}$ с логарифмическими связностями, имеющими одинаковую монодромию. Если все расслоения $E_{t}$ голоморфно тривиальны, то мы имеем изомонодромную деформацию исходной фуксовой системы. Обратно, при некоторых дополнительных условиях на пространство деформации $T$ любая изомонодромная деформация фуксовой системы (3) определяет интегрируемую деформацию $(E, \nabla)$ пары $\left(E^{0}, \nabla^{0}\right)$ такую, что векторное расслоение $E$ голоморфно тривиально, хотя связность $\nabla$ может быть и не логарифмической, в чем мы убедимся в дальнейшем. Это обеспечивается следующей теоремой.

\footnotetext{
${ }^{4}$ Болибрух [15] получил этот результат, рассматривая изомонодромные семейства с пространством деформации $T=D\left(a^{0}\right)$ (см. пример 3), но его доказательство применимо к любому стягиваемому многообразию Штейна $T$.
} 
Теорема 4. Пусть Т - стягиваемое многообразие Штейна. Тогда семейство (13) фуксовых систем изомонодромно тогда и толъко тогда, когда на $X=\overline{\mathbb{C}} \times T$ существует мероморфная матричная дифберенциальная 1-форма $\Omega$ ( с полярным множеством $\left.\bigcup_{i=1}^{n}\left\{z-a_{i}(t)=0\right\}\right)$ такая, что

i) $\Omega=\sum_{i=1}^{n} \frac{B_{i}(t)}{z-a_{i}(t)} d z$ для любого фиксированного $t \in T$;

ii) $d \Omega=\Omega \wedge \Omega$.

ДоКАЗАТЕЛЬСтво. Если семейство (13) изомонодромно, то оно обладает изомонодромной фундаментальной матрицей $Y(z, t)$, которая аналитически зависит от обеих переменных $z$ и $t$, о чем говорилось ранее. Следовательно, $\Omega=d Y \cdot Y^{-1}$ является матричной дифференциальной 1-формой, мероморфной на $X$ и удовлетворяющей условиям i), ii). (Выполнение последнего условия было проверено при доказательстве следствия 1.)

Обратно, если на $X$ существует мероморфная матричная дифференциальная 1-форма $\Omega$, удовлетворяющая условиям i), ii), то она задает интегрируемую связность $\nabla$ в голоморфно тривиальном векторном расслоении $E$ ранга $p$ над $X$ такую, что пара $(E, \nabla)$ является интегрируемой деформацией пары $\left(E^{0}, \nabla^{0}\right)$. Ограничения пары $(E, \nabla)$ на $\overline{\mathbb{C}} \times\{t\}, t \in T$, определяют семейство $\left(E_{t}, \nabla_{t}\right)_{t \in T}$ голоморфно тривиальных векторных расслоений над $\overline{\mathbb{C}}$ с логарифмическими связностями, имеющими одинаковую монодромию. В силу условия і) форма связности $\nabla_{t}$ совпадает с $\sum_{i=1}^{n} \frac{B_{i}(t)}{z-a_{i}(t)} d z$, поэтому семейство (13) изомонодромно. Теорема доказана.

Изомонодромная деформация сохраняет не только монодромию, но и показатели исходной системы (т. е. собственные значения матриц $B_{i}(t)$ не зависят от параметра). В самом деле, множество их возможных значений дискретно вследствие изомонодромности. В то же время, они зависят непрерывно от $t$ как корни многочленов с коэффициентами, голоморфно зависящими от $t$. Поэтому показатели постоянны.

Следует отметить, что 1-форма $\Omega$ из теоремы 4 определена не совсем однозначно. Например, можно рассмотреть другую 1-форму $\Omega^{\prime}=\Omega+(d f) \mathrm{Id}$, где $f$ - голоморфная на $T$ функция. Тогда $\Omega^{\prime}$ и $\Omega$ обе определяют одно и то же семейство, поскольку они совпадают при каждом фиксированном $t \in T$, и $d \Omega^{\prime}=\Omega^{\prime} \wedge \Omega^{\prime}$.

Однако, если монодромия изомонодромного семейства (13) неприводима, то это единственная неопределенность в выборе 1-формы, задающей данное семейство. Точнее, в этом случае матричная дифференциальная 1-форма $\Omega$ из теоремы 4 однозначно определяется семейством с точностью до слагаемого $(d f) f^{-1} \mathrm{Id}$, где $f$ - голоморфная на $T$ функция, не обращающаяся в нуль (см. [2; предложение 15.1]).

Если же монодромия изомонодромного семейства (13) приводима, то оно может иметь нетривиальные симметрии вида

$$
y^{\prime}=\Gamma(z, t) y, \quad \Gamma(z, t)=Y(z, t) C(t) Y^{-1}(z, t),
$$


где $Y(z, t)$ - аналитическая изомонодромная фундаментальная матрица данного семейства и $C(t)$ - голоморфная на $T$ матрица, принадлежащая централизатору группы монодромии семейства. Действительно, в этом случае матрица $\Gamma(z, t)$ мероморфна на $X$ и преобразованная матрица

$$
Y^{\prime}(z, t)=\Gamma(z, t) Y(z, t)=Y(z, t) C(t)
$$

является аналитической изомонодромной фундаментальной матрицей того же семейства (13). Такие симметрии (и только они) задают различные 1-формы

$$
\Gamma \Omega \Gamma^{-1}+(d \Gamma) \Gamma^{-1}
$$

определяющие фиксированное изомонодромное семейство (13).

Обладая критерием из теоремы 4, было бы естественно исследовать, описать и классифицировать матричные дифференциальные 1-формы $\Omega$, ему удовлетворяющие. Это основная цель данного раздела. Как результат мы получим полные описание и классификацию изомонодромных деформаций фуксовых систем, соответствующие, как окажется, и некоторым геометрическим свойствам деформаций.

3.2. Изомонодромные деформации Шлезингера. Мы начнем с изомонодромных деформаций, пространством параметров которых является $T=$ $D\left(a^{0}\right)$ - диск малого радиуса с центром в точке $a^{0}=\left(a_{1}^{0}, \ldots, a_{n}^{0}\right)$ пространства $Z=\mathbb{C}^{n} \backslash \bigcup_{i \neq j}\left\{a_{i}=a_{j}\right\}$ (см. пример 3 ). Рассмотрим семейство

$$
\frac{d y}{d z}=\left(\sum_{i=1}^{n} \frac{B_{i}(a)}{z-a_{i}}\right) y, \quad B_{i}\left(a^{0}\right)=B_{i}^{0}
$$

фуксовых систем, голоморфно зависящее от параметра $a=\left(a_{1}, \ldots, a_{n}\right) \in$ $D\left(a^{0}\right)$. Это семейство задано на пространстве

$$
\left(\overline{\mathbb{C}} \times D\left(a^{0}\right)\right) \backslash \bigcup_{i=1}^{n}\left\{z-a_{i}=0\right\} .
$$

При нахождении мероморфной матричной дифференциальной 1-формы на пространстве $\overline{\mathbb{C}} \times D\left(a^{0}\right)$, совпадающей с $\sum_{i=1}^{n} B_{i}(a) d z /\left(z-a_{i}\right)$ при ограничениях $\left\{a_{i}=\right.$ const $\}$, кажется вполне естественным начать с формы вида

$$
\Omega_{s}=\sum_{i=1}^{n} \frac{B_{i}(a)}{z-a_{i}} d\left(z-a_{i}\right)
$$

Данный анзац имеет долгую историю и известен под названием формы Шлезингера. Такой подход оказывается довольно успешным. Следующее утверждение говорит о том, что для произвольной фуксовой системы можно построить ее изомонодромную деформацию. 
Теорема 5 (Л. Шлезингер [16], [17]). Для любого набора начальных данных $\left\{a_{1}^{0}, \ldots, a_{n}^{0}, B_{1}^{0}, \ldots, B_{n}^{0}\right\}$ существует единственная форма Шлезингера

$$
\Omega_{s}=\sum_{i=1}^{n} \frac{B_{i}(a)}{z-a_{i}} d\left(z-a_{i}\right)
$$

на пространстве $\overline{\mathbb{C}} \times D\left(a^{0}\right)$ такая, что

(i) $B_{i}\left(a^{0}\right)=B_{i}^{0}, i=1, \ldots, n$,

(ii) $d \Omega_{s}=\Omega_{s} \wedge \Omega_{s}$.

Записанное в координатах, условие (ii) выглядит как

$$
d B_{i}=-\sum_{j=1, j \neq i}^{n} \frac{\left[B_{i}, B_{j}\right]}{a_{i}-a_{j}} d\left(a_{i}-a_{j}\right), \quad i=1, \ldots, n,
$$

и известно под названием уравнения Шлезингера, являясь еще одним примером пфаффовой системы (эквивалентность условия $d \Omega_{s}=\Omega_{s} \wedge \Omega_{s}$ уравнению Шлезингера показана, например, в [2; теорема 14.1]). Для того чтобы доказать теорему, достаточно убедиться в интегрируемости этого уравнения. Непосредственно проверяется, что распределение, заданное матричными дифференциальными 1-формами

$$
\Theta_{i}=d B_{i}+\sum_{j=1, j \neq i}^{n} \frac{\left[B_{i}, B_{j}\right]}{a_{i}-a_{j}} d\left(a_{i}-a_{j}\right), \quad i=1, \ldots, n,
$$

удовлетворяет условию интегрируемости Фробениуса (подробнее см. в [4; предложение 5.3 , с. 200] или [2; теорема 14.2]). Таким образом, всякая фуксова система (3) может быть вложена в изомонодромное семейство Шлезингера, семейство вида (14), матрицы-вычеты $B_{i}(a)$ которого удовлетворяют уравнению Шлезингера.

Ниже мы приводим некоторые важные отличительные свойства уравнения Шлезингера (15):

- оно вполне интегрируемо;

- оно допускает гамильтоново [18; дополнение 5], [19; §5] и лаксово [11; пример 3.1] представления;

- оно удовлетворяет свойству Пенлеве (см. п. 4.3);

- при $n \geqslant 4$ и $p=2$ оно эквивалентно системе Гарнье (которая совпадает с шестым уравнением Пенлеве в частном случае $n=4$, см. п. 4.4).

Теорема 5 - это утверждение о локальном существовании изомонодромных деформаций фуксовой системы. Теперь можно задать вопрос о единственности такой деформации и универсальности анзаца Шлезингера: существуют ли другие 1-формы, удовлетворяющие критерию теоремы 4 и определяющие нешлезингеровские деформации, или других возможностей нет? В начальных работах Л.Шлезингера [16], [17] содержится утверждение о том, что семейство (14) изомонодромно тогда и только тогда, когда матрицы $B_{i}(a)$ удовлетворяют уравнению Шлезингера (15). Вообще говоря, это неверно, что мы обсудим чуть позже. Далее же приводятся два результата, касающиеся изомонодромных деформаций Шлезингера. 
Теорема 6 [4; теорема 5.1, с. 196]. Если исходная фуксова система (3) нерезонансна, то ее изомонодромная деформация (14) может быть только шлезингеровской (т.е. матричная дифберенциальная 1-форма $\Omega$, задающая деформачию, может быть выбрана в виде $\Omega=\Omega_{s}$ ).

Теорема 7 (А.А. Болибрух [15], [20]). Если каждая матрица монодромии $G_{i}$ исходной фуксовой системъ (3) удовлетворяет условию

$$
\operatorname{rank}\left(G_{i}-\lambda \mathrm{Id}\right) \geqslant p-1 \quad \forall \lambda \in \mathbb{C},
$$

то ее изомонодромная дебормация (14) может быть толъко илезингеровской.

ЗАмЕчАНИЕ 4. Здесь и в следующем п. 3.3 мы не придаем особого значения тривиальным преобразованиям семейства, которые не зависят от $z$, а зависят только от параметров деформации. Эти преобразования добавляют слагаемое $\sum_{i=1}^{n} C_{i}(a) d a_{i}$ к 1-форме $\Omega$ и переводят 1-формы $\Omega_{s}$ в так называемые ненормализованные формы Шлезингера (и обратно), задающие ненормализованные изомонодромные деформации Шлезингера.

Идея доказательства теорем 6, 7 немедленно следует из конструкции пространства модулей фуксовых систем и интегрируемости уравнения Шлезингеpa. Интегрируемость влечет существование деформации Шлезингера исходной системы. Но возможная альтернатива изомонодромному семейству Шлезингера должна совпадать с ним, поскольку в нерезонансном случае, так же как и в условии теоремы 7 , для любого набора $\left\{a_{1}, \ldots, a_{n}\right\}$ полюсов существует не более одной фуксовой системы с данными полюсами, монодромией и показателями (см. п. 1.2).

Завершая пункт, посвященный изомонодромным деформациям Шлезингера, заметим, что параметр $a=\left(a_{1}, \ldots, a_{n}\right)$ деформации оказывается вполне естественным для них. Действительно, если рассмотреть семейство (13), зависящее от параметра $t \in T$, такое, что $\operatorname{dim} T>n$ и матрицы-вычеты $B_{i}(t)$ удовлетворяют уравнению Шлезингера

$$
d B_{i}(t)=-\sum_{j=1, j \neq i}^{n} \frac{\left[B_{i}(t), B_{j}(t)\right]}{a_{i}(t)-a_{j}(t)} d\left(a_{i}(t)-a_{j}(t)\right)
$$

то для аналитического подмножества

$$
A=\left\{\left(a_{1}(t), \ldots, a_{n}(t)\right)=\text { const }\right\} \subset T
$$

положительной размерности будем иметь $\left.B_{i}\right|_{A}=$ const в силу уравнения Шлезингера. Таким образом, матрицы-вычеты такого семейства можно рассматривать как функции от положения особых точек: $B_{i}=B_{i}(a)$.

3.3. Резонансные изомонодромные деформации. Итак, по-прежнему остается вопрос о существовании изомонодромных деформаций (и матричных дифференциальных 1-форм $\Omega$ из теоремы 4), отличных от шлезингеровских. 
Оказывается, что такие деформации действительно существуют. Первый явный пример нешлезингеровской изомонодромной деформации был построен А. А. Болибрухом (позже появлялись и другие, см. [21], [22]).

ПримеР 4 (А. А. Болибрух [15]). Рассмотрим семейство

$$
\begin{aligned}
\frac{d y}{d z}= & \left(\left(\begin{array}{cc}
1 & 0 \\
-\frac{2 a}{a^{2}-1} & 0
\end{array}\right) \frac{1}{z+a}+\left(\begin{array}{cc}
0 & -6 a \\
0 & -1
\end{array}\right) \frac{1}{z}\right. \\
& \left.+\left(\begin{array}{cc}
2 & 3+3 a \\
\frac{1}{a+1} & -1
\end{array}\right) \frac{1}{z-1}+\left(\begin{array}{cc}
-3 & -3+3 a \\
\frac{1}{a-1} & 2
\end{array}\right) \frac{1}{z+1}\right) y
\end{aligned}
$$

фуксовых систем с особыми точками $a_{1}=-a, a_{2}=0, a_{3}=1, a_{4}=-1$, где $a \in \mathbb{C} \backslash\{0,1,-1\}$ - параметр.

Это семейство изомонодромно, поскольку оно определяется матричной дифференциальной 1-формой

$$
\begin{aligned}
& \Omega=\left(\begin{array}{cc}
1 & 0 \\
-\frac{2 a}{a^{2}-1} & 0
\end{array}\right) \frac{d(z+a)}{z+a}+\left(\begin{array}{cc}
0 & -6 a \\
0 & -1
\end{array}\right) \frac{d z}{z}+\left(\begin{array}{cc}
2 & 3+3 a \\
\frac{1}{a+1} & -1
\end{array}\right) \frac{d(z-1)}{z-1} \\
& +\left(\begin{array}{cc}
-3 & -3+3 a \\
\frac{1}{a-1} & 2
\end{array}\right) \frac{d(z+1)}{z+1}+\left(\begin{array}{cc}
0 & 0 \\
\frac{2 a}{a^{2}-1} & 0
\end{array}\right) \frac{d a}{z+a},
\end{aligned}
$$

которая удовлетворяет условиям теоремы 4. Семейство (16), однако, не является шлезингеровским (ни нормализованным, ни ненормализованным), так как его монодромия неприводима, и поэтому всякая матричная дифференциальная 1-форма, определяющая данное семейство, отличается от $\Omega$ голоморфным слагаемым вида $d f(a) f^{-1}(a) \mathrm{Id}$, где $f$ - голоморфная функция, не обращающаяся в нуль. Таким образом, последнее слагаемое в форме $\Omega$ не может быть упразднено.

Неприводимость монодромии семейства (16) можно объяснить следующим образом. Показатели $\beta_{i}^{j}$ семейства суть

$$
\beta_{1}^{1,2}=0,1, \quad \beta_{2}^{1,2}=0,-1, \quad \beta_{3}^{1,2}=\frac{1}{2} \pm \frac{\sqrt{21}}{2}, \quad \beta_{4}^{1,2}=-\frac{1}{2} \pm \frac{\sqrt{37}}{2}
$$

поэтому собственные значения $\mu_{i}^{j}=e^{2 \pi \sqrt{-1} \beta_{i}^{j}}$ соответствующих матриц монодромии $G_{i}$ суть

$$
\mu_{1}^{1,2}=1, \quad \mu_{2}^{1,2}=1, \quad \mu_{3}^{1,2}=-e^{ \pm \pi \sqrt{-21}}, \quad \mu_{4}^{1,2}=-e^{ \pm \pi \sqrt{-37}} .
$$

Таким образом, $\mu_{1}^{j_{1}} \mu_{2}^{j_{2}} \mu_{3}^{j_{3}} \mu_{4}^{j_{4}} \neq 1$ для любых $j_{1}, j_{2}, j_{3}, j_{4}$, откуда следует неприводимость монодромии (напомним, что для матриц монодромии имеет место соотношение $\left.G_{1} G_{2} G_{3} G_{4}=\mathrm{Id}\right)$. Заметим также, что одна из матриц монодромии $G_{1}, G_{2}$ единичная вследствие теоремы 7 .

Общий вид матричной дифференциальной 1-формы, определяющей изомонодромное семейство (14) фуксовых систем с резонансными особыми точками, 
был получен А. А. Болибрухом [15], [20] (см. также [2; теорема 15.2]). Используемый им аналитический подход может быть применен к произвольному стягиваемому многообразию Штейна $T$. Поэтому мы переходим к изомонодромным деформациям общего вида (13) и описываем матричную дифференциальную 1-форму $\Omega$ из теоремы 4, определяющую такие деформации.

Пусть размерность пространства деформации $T$, которое является стягиваемым многообразием Штейна, равна $s$.

ТЕорема 8. Любая мероморфная матричная дифференииальная 1-форма $\Omega$, задающая изомонодромную дебормачию (13), имеет вид

$$
\Omega=\sum_{i=1}^{n} \frac{B_{i}(t)}{z-a_{i}(t)} d\left(z-a_{i}(t)\right)+\sum_{k=1}^{s}\left(\sum_{i=1}^{n} \sum_{j=1}^{r_{i}} \frac{\gamma_{i j k}(t)}{\left(z-a_{i}(t)\right)^{j}}\right) d t_{k}
$$

в локальных координатах $\left(z, t_{1}, \ldots, t_{s}\right)$ пространства $X=\overline{\mathbb{C}} \times T$, где $\gamma_{i j k}-$ голоморфнье на $T$ матричные функции и $r_{i}$ - максимальный $i$-резонанс семейства (13).

Максимальный $i$-резонанс в приведенной теореме определяется как максимальная разность среди всех целочисленных разностей показателей в точке $a_{i}$ (напомним, что показатели равны собственным значениям матрицы $B_{i}$ ). Такие целочисленные разности всегда существуют для резонансных особых точек. Если точка $a_{i}$ нерезонансна, то $r_{i}=0$ и легко видеть, что теорема 6 является прямым следствием теоремы 8. Более того, любая матричная дифференциальная 1-форма $\Omega$, определяющая нерезонансное изомонодромное семейство, имеет вид $\Omega=\Omega_{s}$ (с точностью до замечания 4).

ДокАЗАТЕЛЬство. Воспользуемся обобщением разложения Левеля (4) на случай изомонодромных семейств, полученным А. А. Болибрухом [20] (см. также [2; теорема 15.1]), - разложением для аналитической изомонодромной фундаментальной матрицы $Y(z, t)$ в окрестности гиперповерхности $\left\{z-a_{i}(t)=0\right\}$. Если матрица монодромии $G_{i}=\operatorname{diag}\left(G_{i}^{1}, \ldots, G_{i}^{m}\right)$, соответствующая матрице $Y\left(z, t^{0}\right)$ в точке $a_{i}^{0}$, блочно-диагональна (в соответствии с разложением в прямую сумму корневых подпространств), то для любого $t^{*} \in T$ найдутся окрестность $D\left(t^{*}\right) \subset T$ и блочно-диагональная матрица $S_{i}(t)=\operatorname{diag}\left(S_{i}^{1}(t), \ldots, S_{i}^{m}(t)\right)$, голоморфно обратимая в $D\left(t^{*}\right)$, такая, что имеет место разложение, аналогичHoe (4):

$$
Y(z, t) S_{i}^{-1}(t)=U_{i}(z, t)\left(z-a_{i}(t)\right)^{\Lambda_{i}}\left(z-a_{i}(t)\right)^{E_{i}(t)},
$$

где матрица $U_{i}(z, t)$ голоморфно обратима в окрестности гиперповерхности $\{z-$ $\left.a_{i}(t)=0\right\}$ и $E_{i}(t)=S_{i}(t) E_{i} S_{i}^{-1}(t)$ - верхнетреугольная матрица.

Пусть $\tilde{Y}(z, t)$ - аналитическая изомонодромная фундаментальная матрица семейства (13) такая, что $\Omega=(d \widetilde{Y}) \widetilde{Y}^{-1}$. Найдется обратимая постоянная матрица $C_{i}$ такая, что матрица монодромии $G_{i}$, соответствующая матрице $\widetilde{Y}(z, t) C_{i}$, блочно-диагональна. Тогда согласно (18) имеем

$$
\widetilde{Y}(z, t) C_{i}=U_{i}(z, t)\left(z-a_{i}(t)\right)^{\Lambda_{i}} S_{i}(t)\left(z-a_{i}(t)\right)^{E_{i}}
$$


в окрестности гиперповерхности $\left\{z-a_{i}(t)=0\right\}$. Поэтому для 1-формы $\Omega$ в этой окрестности выполнено соотношение

$$
\Omega=(d \widetilde{Y}) \widetilde{Y}^{-1}=\left(d \widetilde{Y} C_{i}\right)\left(\widetilde{Y} C_{i}\right)^{-1}=\Omega_{1}+\Omega_{2},
$$

где

$$
\begin{aligned}
\Omega_{1}= & \frac{d U_{i}}{d z} U_{i}^{-1} d\left(z-a_{i}(t)\right)+U_{i}\left(\frac{\Lambda_{i}}{z-a_{i}(t)}+\left(z-a_{i}(t)\right)^{\Lambda_{i}} \frac{E_{i}(t)}{z-a_{i}(t)}\left(z-a_{i}(t)\right)^{-\Lambda_{i}}\right) \\
& \times U_{i}^{-1} d\left(z-a_{i}(t)\right)+\text { голоморфная 1-форма } \\
= & \frac{B_{i}(t)}{z-a_{i}(t)} d\left(z-a_{i}(t)\right)+\text { голоморфная 1-форма, } \\
\Omega_{2}= & U_{i}\left(z-a_{i}(t)\right)^{\Lambda_{i}}\left(d S_{i}\right) S_{i}^{-1}\left(z-a_{i}(t)\right)^{-\Lambda_{i}} U_{i}^{-1} \\
= & \sum_{k=1}^{s}\left(\sum_{j=1}^{r_{i}} \frac{\gamma_{i j k}(t)}{\left(z-a_{i}(t)\right)^{j}}\right) d t_{k}+\text { голоморфная 1-форма, }
\end{aligned}
$$

$\gamma_{i j k}$ - голоморфные матричные функции в координатной окрестности $D\left(t^{*}\right)$ пространства $T$. Вид 1-формы $\Omega_{2}$ следует из блочно-диагональной структуры матриц $\Lambda_{i}, S_{i}$ и определения максимального $i$-резонанса $r_{i}$ семейства (13).

Следовательно, матричная дифференциальная 1-форма

$$
\widetilde{\Omega}=\Omega-\sum_{i=1}^{n} \frac{B_{i}(t)}{z-a_{i}(t)} d\left(z-a_{i}(t)\right)-\sum_{k=1}^{s}\left(\sum_{i=1}^{n} \sum_{j=1}^{r_{i}} \frac{\gamma_{i j k}(t)}{\left(z-a_{i}(t)\right)^{j}}\right) d t_{k}
$$

голоморфна на $\overline{\mathbb{C}} \times D\left(t^{*}\right)$, поэтому $\widetilde{\Omega}=\sum_{k=1}^{s} \gamma_{k}(t) d t_{k}$ (где $\gamma_{k}$ - матричные функции, голоморфные в $\left.D\left(t^{*}\right)\right)$, и мы получаем утверждение теоремы с точностью до замечания 4.

ЗАмЕчАниЕ 5. В доказательстве теоремы 8 возникают матрицы $C_{i} S_{i}^{-1}(t)$, связывающие аналитическую изомонодромную фундаментальную матрицу $\widetilde{Y}(z, t)$ семейства (13) с левелевскими фундаментальными матрицами в точках $a_{i}(t)$. Изомонодромное семейство (13) является шлезингеровским тогда и только тогда, когда эти матрицы связи не зависят от $t$ (см. [2; упражнение 15.3]). На более строгом уровне это утверждение сформулировано в работе [22], где такие деформации (т.е. когда матрицы связи не зависят от $t$ ) названы "isoprincipal".

Частный случай теоремы 8 касается резонансных семейств (14) с пространством деформации $T=D\left(a^{0}\right)$. Как следует из этой теоремы, матричная дифференциальная 1-форма $\Omega$, задающая такую деформацию на пространстве $\overline{\mathbb{C}} \times D\left(a^{0}\right)$, имеет вид

$$
\Omega=\sum_{i=1}^{n} \frac{B_{i}(a)}{z-a_{i}} d\left(z-a_{i}\right)+\sum_{k=1}^{n}\left(\sum_{i=1}^{n} \sum_{j=1}^{r_{i}} \frac{\gamma_{i j k}(a)}{\left(z-a_{i}\right)^{j}}\right) d a_{k}
$$

где $\gamma_{i j k}$ - голоморфные в $D\left(a^{0}\right)$ матричные функции и $r_{i}$ - максимальный $i$-резонанс семейства (14). 
Теорема 8 также приводит к пониманию результата В. А. Побережного [23], изучавшего локальную геометрию пространства деформации $T$ в общем резонансном случае, когда возникают дополнительные параметры деформации помимо особых точек. Предположим, что локально пространство $T$ представляет собой прямое произведение $D\left(a^{0}\right) \times \mathscr{W}$, где $\mathscr{W} \subset \mathscr{M}_{a^{0}, \chi, \exp }^{*}$ непрерывная компонента пространства модулей $\mathscr{M}_{a^{0}}^{*}$, ,exp фуксовых систем с особенностями $a_{1}^{0}, \ldots, a_{n}^{0}$, монодромией $\chi$ и фиксированными показателями, при этом $d=\operatorname{dim} \mathscr{W}>0$. Тогда справедливо следующее утверждение.

Всякая мероморфная матричная дифберенциальная 1-форма $\Omega$ на пространстве $\overline{\mathbb{C}} \times D\left(a^{0}\right) \times \mathscr{W}$, задающая локальную изомонодромную дебормаиию (13), имеет вид

$$
\begin{gathered}
\Omega=\sum_{i=1}^{n} \frac{B_{i}(a, w)}{z-a_{i}} d\left(z-a_{i}\right)+\sum_{k=1}^{n}\left(\sum_{i=1}^{n} \sum_{j=1}^{r_{i}} \frac{\gamma_{i j k}(a, w)}{\left(z-a_{i}\right)^{j}}\right) d a_{k} \\
+\sum_{l=1}^{d}\left(\sum_{i=1}^{n} \sum_{j=1}^{r_{i}} \frac{\beta_{i j l}(a, w)}{\left(z-a_{i}\right)^{j}}\right) d w_{l},
\end{gathered}
$$

где $\gamma_{i j k}, \beta_{i j l}$ - голоморфные на $D\left(a^{0}\right) \times \mathscr{W}$ матричные функции, $w_{1}, \ldots, w_{d}-$ локальные координаты на $\mathscr{W}$ и $r_{i}$-максимальный $i$-резонанс семейства (13).

\section{4. Интегрируемые деформации и свойство Пенлеве}

В этом разделе исследуются особенности уравнений деформаций. Мы описываем множество подвижных особенностей деформации (тета-дивизор) и локальное поведение коэффициентов семейства в окрестности этого множества. Приводятся обобщения классических результатов (установленных для изомонодромных деформаций Шлезингера фуксовых систем), касающихся мероморфного продолжения решений уравнения Шлезингера и глобальной структуры тета-дивизора, на случай интегрируемых деформаций, рассмотренных в теореме 3. Основываясь на работах [24], [25], мы широко используем теорию операторов Фредгольма, кратко представленную в следующем разделе.

4.1. Фредгольмовы операторы. Операторы Фредгольма, о которых мы говорим далее, сыграют свою роль в следующем п. 4.2 при анализе семейств векторных расслоений. Пусть $H$ - сепарабельное комплексное гильбертово пространство. Обозначим через $B(H)$ пространство ограниченных линейных операторов, действующих в $H$.

ОПределЕниЕ 5. Оператор $A \in B(H)$ называется фредголъмовым, если он удовлетворяет следующим свойствам:

- ядро $\operatorname{ker} A$ является конечномерным подпространством в $H$;

- образ $\operatorname{Im} A$ является замкнутым подпространством в $H$ конечной коразмерности

$$
\operatorname{dim} \operatorname{coker} A=\operatorname{dim}(H / \operatorname{Im} A) \text {. }
$$


Множество всех фредгольмовых операторов будем обозначать через $\Phi(H)$. Индекс ind $A$ оператора $A \in \Phi(H)$ определяется как

$$
\text { ind } A=\operatorname{dim} \text { coker } A-\operatorname{dim} \operatorname{ker} A \text {. }
$$

Множество всех обратимых операторов открыто в $B(H)$ и, очевидно, содержится в множестве $\Phi(H)$, но последнее также открыто в $B(H)$ (см., например, [26]). Основные свойства множества $\Phi(H)$ перечислены в следующей теореме. Их доказательства содержатся, например, в книге [27] (см. теорему 1.4 главы 4 и теоремы 2.3, 3.1 главы 5).

ТеОрема 9. Пусть $\mathscr{K}(H)$ - идеал компактных операторов в $B(H)$. Тогда справедливы следующие утверждения:

1) если $A \in \Phi(H)$ и $K \in \mathscr{K}(H)$, то сумма $A+K$ также принадлежит множеству $\Phi(H)$ и индекс не изменяется:

$$
\text { ind } A=\operatorname{ind}(A+K) \text {; }
$$

2) для любого оператора $A \in \Phi(H)$ индекса нуль найдется конечномерный onератор $K \in B(H)$ mакой, что $A+K$ - обратимый оператор;

3) для любых двух операторов $A, B \in \Phi(H)$ произведение $A B$ также принадлежит множеству $\Phi(H)$ и его индекс вычисляется по формуле

$$
\text { ind } A B=\text { ind } A+\text { ind } B \text {. }
$$

Теперь укажем на естественный контекст, относящийся к тем конструкциям данной работы, в которых встречаются фредгольмовы операторы. Пусть $S^{1}-$ единичная окружность в комплексной плоскости и $H=L^{2}\left(S^{1}, \mathbb{C}^{p}\right)$ - гильбертово пространство $\mathbb{C}^{p}$-значных функций, интегрируемых вместе с квадратом. Элементы пространства $H$ представляются рядами Фурье

$$
h=\sum_{n \in \mathbb{Z}} b_{n} e^{i n \varphi}, \quad b_{n} \in \mathbb{C}^{p} .
$$

Пространство $H$ разлагается в прямую сумму $H_{+} \oplus H_{-}$, где

$$
H_{+}=\left\{\sum_{n \geqslant 0} b_{n} z^{n}\right\}
$$

- замыкание относительно $L^{2}$-нормы подпространства непрерывных на $S^{1}$ функций, продолжающихся голоморфно в единичный диск, а

$$
H_{-}=\left\{\sum_{n<0} b_{n} z^{n}\right\}
$$

- замыкание относительно той же нормы подпространства непрерывных на $S^{1}$ функций, которые продолжаются голоморфно во внешность единичной окружности и равны нулю в бесконечности.

Обозначим через $\Gamma(U)$ группу всех голоморфных отображений $\gamma$ связной окрестности $U$ окружности $S^{1}$ в $\mathrm{GL}(p, \mathbb{C})$, и пусть Г обозначает ее индуктивный предел относительно $U$. Эта группа состоит из функций перехода для 
голоморфных векторных расслоений ранга $p$ над $\overline{\mathbb{C}}$. Элементы $\gamma \in \Gamma$ действуют на пространстве $H$ умножением слева как линейные операторы $M_{\gamma}$.

Для $\gamma \in \Gamma$ предста́вим оператор $M_{\gamma}$ в соответствии с разложением $H=$ $H_{+} \oplus H_{-}$как

$$
M_{\gamma}=\left(\begin{array}{ll}
A & B \\
C & D
\end{array}\right) .
$$

Покажем, что $A$ является оператором Фредгольма.

Согласно теореме Биркгофа-Гротендика [28], [29], каждое голоморфное векторное расслоение $E$ ранга $p$ над $\overline{\mathbb{C}}$ является прямой суммой линейных расслоений,

$$
E \cong \mathscr{O}\left(m_{1}\right) \oplus \cdots \oplus \mathscr{O}\left(m_{p}\right), \quad m_{i} \in \mathbb{Z}
$$

т. е. матричная функция $\gamma(z)$ разлагается в произведение

$$
\gamma(z)=\gamma_{-}(z)\left(\begin{array}{ccc}
z^{m_{1}} & & 0 \\
& \ddots & \\
0 & & z^{m_{p}}
\end{array}\right) \gamma_{+}(z)=\gamma_{-}(z) \gamma_{0}(z) \gamma_{+}(z)
$$

где $\gamma_{+}-$элемент группы $Г$, который продолжается голоморфно в нуль, и $\gamma_{-}-$ элемент группы $Г$, который продолжается голоморфно в бесконечность и удовлетворяет условию $\gamma_{-}(\infty)=\mathrm{Id}$. Поэтому $M_{\gamma}=M_{\gamma_{-}} M_{\gamma_{0}} M_{\gamma_{+}}$, при этом ограничения $\left.M_{\gamma_{-}}\right|_{H_{-}}$и $\left.M_{\gamma_{+}}\right|_{H_{+}}$являются изоморфизмами подпространств $H_{-}$и $H_{+}$ соответственно. Следовательно, операторы $M_{\gamma_{-}}$и $M_{\gamma_{+}}$в соответствии с разложением $H=H_{+} \oplus H_{-}$могут быть представлены в виде

$$
M_{\gamma_{-}}=\left(\begin{array}{cc}
A_{-} & 0 \\
C_{-} & D_{-}
\end{array}\right), \quad M_{\gamma_{+}}=\left(\begin{array}{cc}
A_{+} & B_{+} \\
0 & D_{+}
\end{array}\right),
$$

где $A_{-}, A_{+}: H_{+} \rightarrow H_{+}-$изоморфизмы ${ }^{5}$ и, значит, являются фредгольмовыми операторами индекса нуль. Представляя оператор $M_{\gamma_{0}}$ в соответствии с разложением $H=H_{+} \oplus H_{-}$в виде

$$
M_{\gamma_{0}}=\left(\begin{array}{ll}
A_{0} & B_{0} \\
C_{0} & D_{0}
\end{array}\right)
$$

получаем соотношение $A=A_{-} A_{0} A_{+}$. То, что оператор $A_{0}$ фредгольмов, можно проверить следующим образом.

Пусть $e_{1}, \ldots, e_{p}-$ стандартный базис пространства $\mathbb{C}^{p}$. Из явного вида матричной функции $\gamma_{0}(z)$ следует, что $M_{\gamma_{0}}\left(e_{i} z^{n}\right)=e_{i} z^{n+m_{i}}$, поэтому

$$
\operatorname{ker} A_{0}=\left\langle e_{i} z^{n} \mid m_{i}<0,0 \leqslant n<-m_{i}\right\rangle, \quad \operatorname{Im} A_{0}=\left\langle e_{i} z^{n} \mid n \geqslant m_{i} \geqslant 0\right\rangle .
$$

Таким образом,

$$
\operatorname{dim} \operatorname{ker} A_{0}=-\sum_{\left\{i: m_{i}<0\right\}} m_{i} \quad \text { и } \quad \operatorname{dim} \operatorname{coker} A_{0}=\sum_{\left\{i: m_{i}>0\right\}} m_{i} .
$$

\footnotetext{
${ }^{5}$ Несложно проверить, что для любого элемента $h_{+} \in H_{+}$уравнение $A_{-}\left(x_{+}\right)=h_{+}$имеет единственное решение $x_{+} \in H_{+}$. Это решение - проекция элемента $\gamma_{-}^{-1} h_{+} \in H$ на подпространство $H_{+}$.
} 
Это значит, что оператор $A_{0}$ фредгольмов индекса $\sum_{i=1}^{p} m_{i}$. Тогда, вследствие теоремы 9 , то же верно и для оператора $A=A_{-} A_{0} A_{+}$. Заметим, что индекс ind $A=\sum_{i=1}^{p} m_{i}$ оператора $A$ равен степени голоморфного векторного расслоения, заданного функцией перехода $\gamma(z)$.

4.2. Нетривиальность деформируемого расслоения. Мы возвращаемся к интегрируемым деформациям $(E, \nabla)$ пары $\left(E^{0}, \nabla^{0}\right)$, построенным во втором разделе. Напомним, что исходное векторное расслоение $E^{0}$ ранга $p$ над $\overline{\mathbb{C}}$ было голоморфно тривиальным. Следовательно, ограничение $\left.E\right|_{\overline{\mathbb{C}} \times\left\{t^{0}\right\}}$ также тривиально. Можно поинтересоваться, будет ли тривиальным расслоение $E_{t}=\left.E\right|_{\overline{\mathbb{C}} \times\{t\}}$ при изменении параметра деформации $t \in T$. Рассмотрим подмножество $\Theta$ пространства $T$, на котором это свойство нарушается, т. е.

$$
\Theta=\left\{t \in T \mid E_{t} \text { нетривиально }\right\} .
$$

ПРЕДЛОЖЕНИЕ 1 (см. также [24] и [30]). Множество $\Theta$ или пусто, или является аналитическим подмножеством $T$ коразмерности один.

ДокАЗАТЕЛьство. Выберем действительные числа $\rho_{1}$ и $\rho_{2}$ такие, что $0<$ $\rho_{1}<1<\rho_{2}$, и рассмотрим множества

$$
D_{1}=\left\{z \in \overline{\mathbb{C}}|| z \mid>\rho_{1}\right\}, \quad D_{2}=\left\{z \in \overline{\mathbb{C}}|| z \mid<\rho_{2}\right\} .
$$

Возьмем также открытую окрестность $U$ точки $t \in T$, которая является стягиваемым штейновым многообразием. Тогда векторные расслоения $\left.E\right|_{D_{1} \times U}$ и $\left.E\right|_{D_{2} \times U}$ голоморфно тривиальны. (Если $T=\widetilde{Z}$, как в теореме 3 , то можно взять $U=\widetilde{Z}$.) Пусть $\left\{f_{1}, \ldots, f_{p}\right\}$ и $\left\{g_{1}, \ldots, g_{p}\right\}$ - базисы голоморфных сечений расслоений $\left.E\right|_{D_{1} \times U}$ и $\left.E\right|_{D_{2} \times U}$ соответственно. Тогда имеется голоморфная функция перехода

$$
S:\left(D_{1} \cap D_{2}\right) \times U \rightarrow \operatorname{GL}(p, \mathbb{C}),
$$

которая выражает $\left\{f_{i}\right\}$ через $\left\{g_{i}\right\}$, т. е. $\left(g_{1}, \ldots, g_{p}\right)=\left(f_{1}, \ldots, f_{p}\right) S$. Возьмем произвольную точку $t \in U$. Из теоремы Биркгофа-Гротендика заключаем о существовании голоморфных отображений

$$
S_{+}(\cdot, t): D_{2} \rightarrow \mathrm{GL}(p, \mathbb{C}), \quad S_{-}(\cdot, t): D_{1} \rightarrow \mathrm{GL}(p, \mathbb{C})
$$

таких, что вышеприведенное семейство (относительно $U$ ) элементов группы $\Gamma$ представляется в виде

$$
S(z, t)=S_{-}^{-1}(z, t) \operatorname{diag}\left(z^{m_{1}}, \ldots, z^{m_{p}}\right) S_{+}(z, t),
$$

где $m_{1} \geqslant \cdots \geqslant m_{p}$ - целые и можно считать, что $S_{-}(\infty, t)=\mathrm{Id}$. Расслоение $E_{t}$ голоморфно тривиально тогда и только тогда, когда все $m_{i}$ равны нулю, т. е. когда для всех $z \in D_{1} \cap D_{2}$ имеет место соотношение

$$
S(z, t)=S_{-}^{-1}(z, t) S_{+}(z, t)
$$


Каждому члену из разложения (21) ставится в соответствие ограниченный линейный оператор на пространстве $H=L^{2}\left(S^{1}, \mathbb{C}^{p}\right)$. Как мы видели в п. 4.1, умножение на $S(\cdot, t)$ определяет при фиксированном $t \in U$ ограниченный линейный оператор $\mathbb{S}(t)$ на пространстве $H$, который в соответствии с разложением $H=H_{+} \oplus H_{-}$представляется в виде

$$
\mathbb{S}(t)=\left(\begin{array}{ll}
A(t) & B(t) \\
C(t) & D(t)
\end{array}\right)
$$

где $A(t): H_{+} \rightarrow H_{+}-$фредгольмов оператор индекса $\sum_{i=1}^{p} m_{i}$. При этом отображение $t \mapsto A(t)$ голоморфно, поскольку матричная функция $S(z, t)$ голоморфно зависит от $t$.

Далее, все сомножители из правой части соотношения (21) определяют ограниченные линейные операторы на пространстве $H$, которые в соответствии с разложением $H=H_{+} \oplus H_{-}$представляются в виде

$$
\left(\begin{array}{cc}
A_{-} & 0 \\
C_{-} & D_{-}
\end{array}\right), \quad\left(\begin{array}{cc}
A_{0} & B_{0} \\
C_{0} & D_{0}
\end{array}\right) \quad \text { и } \quad\left(\begin{array}{cc}
A_{+} & B_{+} \\
0 & D_{+}
\end{array}\right)
$$

соответственно, поэтому

$$
A(t)=A_{-} A_{0} A_{+} .
$$

Поскольку операторы $A_{-}$и $A_{+}$обратимы, обратимость оператора $A(t)$ эквивалентна обратимости оператора $A_{0}$, а, как видно из п. 4.1, это имеет место тогда и только тогда, когда все $m_{i}$ равны нулю, т. е. когда расслоение $E_{t}$ голоморфно тривиально и справедливо разложение (22).

Поскольку множество $U$ связно и оператор $A\left(t^{0}\right)$ обратим (расслоение $E_{t^{0}}$ голоморфно тривиально), то ind $A(t)=\operatorname{deg} E_{t}=0$ для всех $t \in U$ (другими словами, степень расслоений $E_{t}, t \in U$, не меняется в ходе интегрируемой деформации $\left.{ }^{6}\right)$. А так как оператор $A(t)$ фредгольмов, то из предыдущего факта следует (см. теорему 9), что для любой точки $t \in U$ найдется конечномерный оператор $K(t) \in B\left(H_{+}\right)$такой, что оператор $A(t)+K(t)$ обратим. Локально можно даже выбрать $K(t)=K$ независимым от $t$. В самом деле, если оператор $A\left(t^{1}\right)+K\left(t^{1}\right)$ обратим, то отображение $t \mapsto A(t)+K\left(t^{1}\right)$ является непрерывным отображением из $U$ в $\Phi\left(H_{+}\right)$. Поскольку множество обратимых операторов открыто в $\Phi\left(H_{+}\right)$, то операторы $A(t)+K\left(t^{1}\right)$ обратимы при всех $t$, близких к $t^{1}$.

${ }^{6}$ Действительно, из соотношения (21) следует, что

$$
\frac{d_{z} \operatorname{det} S(z, t)}{\operatorname{det} S(z, t)}=\frac{\sum_{i=1}^{p} m_{i}}{z} d z+\frac{d_{z} \operatorname{det} S_{+}(z, t)}{\operatorname{det} S_{+}(z, t)}-\frac{d_{z} \operatorname{det} S_{-}(z, t)}{\operatorname{det} S_{-}(z, t)}
$$

поэтому

$$
\operatorname{deg} E_{t}=\sum_{i=1}^{p} m_{i}=\int_{|z|=1} \frac{d_{z} \operatorname{det} S(z, t)}{\operatorname{det} S(z, t)}
$$

(дифференциальные 1-формы $\frac{d_{z} \operatorname{det} S_{+}(z, t)}{\operatorname{det} S_{+}(z, t)}$ и $\frac{d_{z} \operatorname{det} S_{-}(z, t)}{\operatorname{det} S_{-}(z, t)}$ голоморфны в $D_{2}$ и $D_{1}$ соответственно, поэтому интегралы от них по единичной окружности равны нулю). Таким образом, целочисленная функция $\operatorname{deg} E_{t}$ голоморфно зависит от параметра $t$, следовательно, она постоянна. 
Таким образом, имеем

$$
A(t)(A(t)+K)^{-1}=\mathrm{Id}-K(A(t)+K)^{-1},
$$

т. е. для любой точки $t_{\alpha} \in U$ найдутся ее открытая окрестность $U_{\alpha}$ и голоморфное отображение

$$
Q_{\alpha}: U_{\alpha} \rightarrow \operatorname{Aut}\left(H_{+}\right)
$$

такие, что оператор $A(t) Q_{\alpha}^{-1}(t)$ - Id является конечномерным. Согласно [29], всякий ограниченный линейный оператор вида Id $+\{$ конечномерный $\}$ обладает корректно определенным детерминантом, и такой оператор обратим тогда и только тогда, когда его детерминант отличен от нуля. Следовательно, локально в окрестности $U_{\alpha}$ имеем

$$
U_{\alpha} \cap \Theta=\left\{t \in U_{\alpha} \mid \tau_{\alpha}(t):=\operatorname{det}\left(A(t) Q_{\alpha}^{-1}(t)\right)=0\right\},
$$

что доказывает предложение.

На каждом непустом пересечении $U_{\alpha} \cap U_{\beta}$ имеем

$$
\left(A(t) Q_{\alpha}^{-1}(t)\right)\left(Q_{\alpha}(t) Q_{\beta}^{-1}(t)\right)=\left(A(t) Q_{\beta}^{-1}(t)\right) .
$$

Поскольку операторы $A(t) Q_{\alpha}^{-1}(t), A(t) Q_{\beta}^{-1}(t)$ имеют вид $\mathrm{Id}+\{$ конечномерный $\}$, такой же вид имеет и обратимый оператор $Q_{\alpha}(t) Q_{\beta}^{-1}(t)$. Следовательно, он обладает не обращающимся в нуль детерминантом и функции $\tau_{\alpha}, \tau_{\beta}$ отличаются умножением на не обращающуюся в нуль голоморфную функцию

$$
\operatorname{det}\left(Q_{\alpha}(t) Q_{\beta}^{-1}(t)\right) .
$$

Набор таких функций задает линейное расслоение над $U$, которое голоморфно тривиально, поскольку $U$ является стягиваемым многообразием Штейна. Следовательно, локальные функции $\tau_{\alpha}$, описывающие множество $\Theta$, склеиваются в глобальную голоморфную функцию $\tau: U \rightarrow \mathbb{C}$, для которой множество $U \cap \Theta$ является множеством нулей. Таким образом, для пространств деформации $T=\widetilde{Z}$ или $T=D\left(a^{0}\right)$ найдется глобальная голоморфная функция $\tau: T \rightarrow \mathbb{C}$ такая, что множество $\Theta$ является множеством ее нулей. Тогда аналогичные функции для остальных интегрируемых деформаций, построенных в разделе 2 (с пространством деформации $T=D\left(a^{0}\right) \times \mathscr{W}$, где $\mathscr{W}-$ связное комплексное многообразие, а также с произвольным односвязным пространством деформации $T)$, будут иметь вид $\tau$ ○ $f$, где $f$ - соответствующее отображение $D\left(a^{0}\right) \times \mathscr{W} \rightarrow D\left(a^{0}\right)$ или $T \rightarrow \widetilde{Z}$. Итак, получаем следующее утверждение.

СледСтвиЕ 2. Для каждой из интегрируемых деформаций $(E, \nabla)$, построенных в разделе 2 , и соответствующего пространства деформации $T$ найдется глобальная голоморфная функиия $\tau: T \rightarrow \mathbb{C}$ такая, что

$$
\Theta=\{t \in T \mid \tau(t)=0\}
$$

Обычно функцию $\tau$ называют (глобальной) $\tau$-функиией интегрируемой деформации $(E, \nabla)$. 
Исследуем теперь характер зависимости матриц $S_{+}(z, t)$ и $S_{-}(z, t)$ от переменной $t$, выразив разложение $(22)$ в терминах оператора $A(t)$. Если $t \notin \Theta$, то определим матрицу $\Sigma_{+}(z, t)$ соотношением

$$
\Sigma_{+}(z, t)=A^{-1}(t) \mathrm{Id},
$$

в котором столбцы единичной матрицы размера $p \times p$ понимаются как элементы пространства $H_{+}$и оператор $A^{-1}(t)$ действует на них естественным образом. Следовательно, матричная функция $\Sigma_{+}$голоморфна (по $z$ ) в окрестности диска $\{z \in \mathbb{C}|| z \mid \leqslant 1\}$. Из определения $\Sigma_{+}(z, t)$ видно, что

$$
A(t) \Sigma_{+}(z, t)=\mathrm{Id}
$$

где оператор $A(t)$ действует на столбцы матрицы $\Sigma_{+}(z, t)$ как на элементы пространства $H_{+}$. Таким образом,

$$
S(z, t) \Sigma_{+}(z, t)=\operatorname{Id}+\Sigma_{-}(z, t)
$$

где $\Sigma_{-}-$матричная функция, голоморфная (по $z$ ) в окрестности множества $\{z \in \overline{\mathbb{C}}|| z \mid \geqslant 1\}$, и $\Sigma_{-}(\infty, t)=0$, поскольку ее столбцы являются элементами пространства $H_{-}$. Учитывая то, что голоморфными функциями на $\overline{\mathbb{C}}$ могут быть только константы, и используя соотношение $S(z, t)=S_{-}^{-1}(z, t) S_{+}(z, t)$, получаем

$$
S_{+}(z, t) \Sigma_{+}(z, t)=S_{-}(z, t)\left\{\operatorname{Id}+\Sigma_{-}(z, t)\right\}=S_{-}(\infty, t)\left\{\operatorname{Id}+\Sigma_{-}(\infty, t)\right\}=\operatorname{Id} .
$$

Из этого следует обратимость матриц $\Sigma_{+}(z, t)$ и $\mathrm{Id}+\Sigma_{-}(z, t)$. А так как они голоморфно зависят от $t \in U \backslash \Theta$, то матрицы $S_{+}(z, t)$ и $S_{-}(z, t)$ обладают этим же свойством. Покажем теперь, что последние матрицы мероморфны вдоль $D_{2} \times \Theta$ и $D_{1} \times \Theta$ соответственно.

Из [29] заключаем, что оператор, обратный к оператору вида Id $+F$, где $F-$ конечномерный оператор, принадлежащий пространству $B\left(H_{+}\right)$, задается формулой

$$
(\mathrm{Id}+F)^{-1}=\frac{1}{\operatorname{det}(\mathrm{Id}+F)} R(F) .
$$

Здесь оператор $R(F) \in B\left(H_{+}\right)$определен при помощи ряда

$$
R(F)=\sum_{n=0}^{\infty} R_{n}(F),
$$

где $R_{0}(F)=\mathrm{Id}$ и для каждого $n \geqslant 1$ оператор $R_{n}(F) \in B\left(H_{+}\right)$определяется как

$$
R_{n}(F)=R_{n}(F, \ldots, F),
$$

с использованием $R_{n}$ - мультилинейного отображения из $B_{\mathrm{f}}\left(H_{+}\right)^{n}, n$-кратного декартова произведения пространства $B_{\mathrm{f}}\left(H_{+}\right) \subset B\left(H_{+}\right)$конечномерных операторов, в пространство $B\left(H_{+}\right)$. Данное мультилинейное отображение задается спариванием

$$
\operatorname{Tr}\left(F_{0} \circ R_{n}\left(F_{1}, \ldots, F_{n}\right)\right)=(n+1) \operatorname{Tr}\left(F_{1} \wedge \cdots \wedge F_{n} \wedge F_{0}\right) \quad \forall F_{0} \in B_{\mathrm{f}}\left(H_{+}\right),
$$


где $\operatorname{Tr}$ обозначает след участвующих здесь операторов. Заметим, что сумма $(23)$ конечна при любом $F \in B_{\mathrm{f}}\left(H_{+}\right)$, поскольку $n$-я внешняя степень такого оператора $F$ равна нулю при $n>\operatorname{dim} \operatorname{Im} F$. Кроме того, если оператор $F$ голоморфно зависит от параметра $t$, то оператор $R(F)$ также обладает этим свойством. Используем приведенные общие факты в нашем контексте. Пусть, как и ранее,

$$
Q_{\alpha}: U_{\alpha} \rightarrow \operatorname{Aut}\left(H_{+}\right)
$$

- голоморфное отображение такое, что оператор $F_{\alpha}(t):=A(t) Q_{\alpha}^{-1}(t)-\mathrm{Id}$ является конечномерным. Тогда имеем

$$
\begin{aligned}
\Sigma_{+}(z, t) & =A^{-1}(t) \operatorname{Id}=Q_{\alpha}^{-1}(t)\left(A(t) Q_{\alpha}^{-1}(t)\right)^{-1} \operatorname{Id}=Q_{\alpha}^{-1}(t)\left(\operatorname{Id}+F_{\alpha}(t)\right)^{-1} \operatorname{Id} \\
& =\frac{1}{\operatorname{det}\left(\operatorname{Id}+F_{\alpha}(t)\right)} Q_{\alpha}^{-1}(t) R\left(F_{\alpha}(t)\right) \operatorname{Id} .
\end{aligned}
$$

Из этого следует, что отображение $t \mapsto \tau_{\alpha}(t) \Sigma_{+}(z, t)$ голоморфно в $U_{\alpha}$ и, таким образом, матрица $\Sigma_{+}(z, t)$ мероморфна (по $t$ ) вдоль $\Theta$. Это же справедливо и для ее обратной $S_{+}(z, t)$. Матрица Id $+\Sigma_{-}(z, t)$ по определению равна $S(z, t) \Sigma_{+}(z, t)$. Следовательно, обратная $S_{-}(z, t)$ к матрице Id $+\Sigma_{-}(z, t)$ также мероморфна (по $t$ ) вдоль $\Theta$. Итак, получаем следующее утверждение.

ПредложениЕ 2 (см. также [24] и [30]). Пусть T-связное комплексное многообразие, $t^{0} \in T u$

$$
S:\left(D_{1} \cap D_{2}\right) \times T \rightarrow \mathrm{GL}(p, \mathbb{C})
$$

- голоморфное отображение такое, что

$$
S\left(z, t^{0}\right)=F_{-}^{-1}(z) F_{+}(z)
$$

где $F_{-}$и $F_{+}-$голоморфно обратимые матричные функиии в $D_{1}$ и $D_{2}$ coответственно. Тогда найдется подмножество $\Theta \subset T$, которое либо пусто, либо является аналитическим подмножеством коразмерности один, а также найдутся голоморфные отображения

$$
S_{+}: D_{2} \times(T \backslash \Theta) \rightarrow \mathrm{GL}(p, \mathbb{C}), \quad S_{-}: D_{1} \times(T \backslash \Theta) \rightarrow \mathrm{GL}(p, \mathbb{C})
$$

такие, что:

1) $S(z, t)=S_{-}^{-1}(z, t) S_{+}(z, t) u S_{-}(\infty, t)=\mathrm{Id}$;

2) отображения $S_{+} u S_{-}$мероморфны вдоль $D_{2} \times \Theta u D_{1} \times \Theta$ соответственно.

Обратимся еще раз к доказательству предложения 1. Рассмотрим открытое стягиваемое многообразие Штейна $U \subset T$ и базисы $\left\{f_{i}\right\},\left\{g_{i}\right\}$. Тогда голоморфные на $\overline{\mathbb{C}} \times(U \backslash \Theta)$ сечения

$$
\left(h_{1}, \ldots, h_{p}\right)=\left(f_{1}, \ldots, f_{p}\right) S_{-}^{-1}=\left(g_{1}, \ldots, g_{p}\right) S_{+}^{-1}
$$

будут образовывать базис глобальных сечений (линейно независимых в каждой точке) расслоения, эквивалентного расслоению $\left.E\right|_{\overline{\mathbb{C}} \times(U \backslash \Theta)}$. Таким образом, 
расслоение $\left.E\right|_{\overline{\mathbb{C}} \times(T \backslash \Theta)}$ голоморфно тривиально в случае интегрируемой деформации $(E, \nabla)$ с пространством деформации $T=\widetilde{Z}$ или $T=D\left(a^{0}\right)$. Поскольку остальные интегрируемые деформации из раздела 2 получались как обратные прообразы последней, то для них справедливо аналогичное утверждение.

СлеДСТВИЕ 3. Для каждой из интегрируемых деформаций $(E, \nabla)$, nостроенных в разделе 2, векторное расслоение $E$ голоморфно тривиально над $\overline{\mathbb{C}} \times(T \backslash \Theta)$.

4.3. Мероморфное продолжение. Рассмотрим открытое стягиваемое многообразие Штейна $U \subset T$ и интегрируемую связность $\nabla(\infty)$ в голоморфно тривиальном векторном расслоении $\left.E\right|_{\{\infty\} \times U}$ из замечания 3 на с. 80 . Пусть $\left\{s_{1}, \ldots, s_{p}\right\}$ является базисом горизонтальных сечений этой связности. Выберем теперь базис $\left\{f_{1}, \ldots, f_{p}\right\}$ голоморфных сечений ограничения $\left.E\right|_{D_{1} \times U}$ такой, что $\left.f_{j}\right|_{\{\infty\} \times U}=s_{j}$. Пусть также $\left\{g_{1}, \ldots, g_{p}\right\}$ - базис голоморфных сечений ограничения $\left.E\right|_{D_{2} \times U}$ и $S(z, t)$, как в предыдущем пункте, - матрица перехода, связывающая $\left\{g_{j}\right\}$ и $\left\{f_{j}\right\}$.

Согласно предложению 2 матрица перехода $S(z, t)$ разлагается в области $\left(D_{1} \cap D_{2}\right) \times(U \backslash \Theta)$ в произведение $S=S_{-}^{-1} S_{+}$. Тогда базис $\left\{h_{j}\right\}$ голоморфных сечений из следствия 3 состоит из тривиальных продолжений базиса $\left\{s_{j}\right\}$. Пусть $\Omega$ является формой связности $\nabla$ в базисе $\left\{h_{j}\right\}$. Из формулы (12) видно, что в окрестности гиперповерхности

$$
Y_{i}=\left\{(z, t) \in \overline{\mathbb{C}} \times U \mid z-a_{i}(t)=0\right\}
$$

матричная дифференциальная 1-форма $\Omega$ выглядит как

$$
\Omega=\sum_{l \geqslant 1} \frac{B_{i l}(t)}{\left(z-a_{i}(t)\right)^{l}} d\left(z-a_{i}(t)\right)+w_{i},
$$

где $w_{i}$ - голоморфная матричная дифференциальная 1-форма возле $Y_{i}$ и матрицы $B_{i l}$ голоморфны в $U \backslash \Theta$. Определим теперь матричную дифференциальную 1-форму

$$
\Omega_{f}=\sum_{i=1}^{n} \sum_{l \geqslant 1} \frac{B_{i l}(t)}{\left(z-a_{i}(t)\right)^{l}} d\left(z-a_{i}(t)\right),
$$

мероморфную на $\overline{\mathbb{C}} \times(U \backslash \Theta)$. Тогда $\Omega_{\infty}=\Omega-\Omega_{f}$ является матричной дифференциальной 1-формой, голоморфной на $\overline{\mathbb{C}} \times(U \backslash \Theta)$. Поэтому она зависит только от $t$. Из определения связности $\nabla(\infty)$ непосредственно следует, что $\Omega_{\infty}$ - форма связности $\nabla(\infty)$ в базисе $\left\{s_{j}\right\}$. А так как сечения $s_{j}$ горизонтальны, получаем $\Omega_{\infty}=0$, т. е. $\Omega=\Omega_{f}$.

Поскольку пара $\left(E_{t^{0}}, \nabla_{t^{0}}\right)$ изоморфна паре $\left(E^{0}, \nabla^{0}\right)$, то можно сделать вывод о существовании матрицы $C \in \mathrm{GL}(p, \mathbb{C})$ такой, что

$$
B_{i l}\left(t^{0}\right)=C B_{i l}^{0} C^{-1}
$$

при всех $i, l$. Поэтому (применив линейное преобразование к базису $\left\{h_{j}\right\}$ ) можно предполагать, что матрицы $B_{i l}(t)$ удовлетворяют начальным условиям $B_{i l}\left(t^{0}\right)=B_{i l}^{0}$ при всех $i, l$. Для того чтобы понять, почему эти матрицы 
мероморфны на $U$, рассмотрим формы $\Omega_{1}$ и $\Omega_{2}$ связности $\nabla$ в базисах $\left\{f_{j}\right\}$ и $\left\{g_{j}\right\}$ соответственно. Тогда $\Omega_{1}, \Omega_{2}$ голоморфны на $\left(D_{1} \times U\right) \backslash Y,\left(D_{2} \times U\right) \backslash Y$ и мероморфны вдоль $Y=Y_{1} \cup \cdots \cup Y_{n}$. Они связаны с формой $\Omega$ следующим образом:

$$
\begin{array}{ll}
\Omega=S_{-} \Omega_{1} S_{-}^{-1}+\left(d S_{-}\right) S_{-}^{-1} & \text { на } D_{1} \times(U \backslash \Theta), \\
\Omega=S_{+} \Omega_{2} S_{+}^{-1}+\left(d S_{+}\right) S_{+}^{-1} & \text { на } D_{2} \times(U \backslash \Theta) .
\end{array}
$$

Принимая во внимание предложение 2, можно подвести следующий промежуточный итог данного пункта.

Рассмотрим мероморфную линейную систему (1) с особенностями $a_{1}^{0}, \ldots, a_{n}^{0}$ в комплексной плоскости, так что все матрицы $B_{\infty l}^{0}$ равны нулю и выполнено соотношение (2). Интерпретируя данную систему как уравнение горизонтальных сечений мероморфной связности $\nabla^{0}$ в голоморфно тривиальном векторном расслоении $E^{0}$, можно построить интегрируемую деформацию $(E, \nabla)$ пары $\left(E^{0}, \nabla^{0}\right)$ согласно теореме 3 (и замечанию 2 ).

ТЕОРема 10. Каждая из интегрируемых деформаций $(E, \nabla)$, построенных в разделе 2, обладает следующими свойствами:

1) для всякой точки $t^{0} \in T$ найдется ее окрестность $V_{0}$ такая, что ограничение $\left.E\right|_{\overline{\mathbb{C}} \times V_{0}}$ голоморфно тривиально, при этом существует базис сечении данного ограничения, в котором форма $\Omega$ связности $\nabla$ имеет вид

$$
\Omega=\sum_{i=1}^{n} \sum_{l \geqslant 1} \frac{B_{i l}(t)}{\left(z-a_{i}(t)\right)^{l}} d\left(z-a_{i}(t)\right),
$$

$u B_{i l}\left(t^{0}\right)=B_{i l}^{0}$ nри всех $i, l$;

2) решение $\left\{B_{i l}(t)\right\}$ уравнения интегрируемости продолжсается голоморфно на $T \backslash \Theta$ и оказывается мероморфным вдоль $\Theta$.

Для интегрируемой деформации, построенной в теореме 3, вышеприведенная теорема обеспечивает мероморфное продолжение решения $\left\{B_{i l}\right\}$ на пространство $\widetilde{Z}$. В случае, когда связность $\nabla^{0}$ логарифмическая, получается знаменитая теорема Б. Мальгранжа.

Теорема 11 (Б. Мальгранж [24], [19]). Пусть $\left\{B_{1}(a), \ldots, B_{n}(a)\right\}-$ локальное решение уравнения Шлезингера (15) с произвольными начальными условиями $B_{i}\left(a^{0}\right)=B_{i}^{0}$. Тогда матрицы $B_{i}(a)$ продолжаются на универсальное накрытие $\widetilde{Z}$ пространства $\mathbb{C}^{n} \backslash \bigcup_{i \neq j}\left\{a_{i}=a_{j}\right\}$ как мероморфные функции.

Утверждение данной теоремы известно под названием свойства Пенлеве уравнения Шлезингера. Обычно говорят, что дифференциальное уравнение (или система дифференциальных уравнений) обладает свойством Пенлеве, если 
его подвижными особенностями ${ }^{7}$ могут быть только полюсы или, что эквивалентно, все решения продолжаются мероморфно на универсальное накрытие пространства независимой переменной, проколотого в неподвижных особенностях.

Полярное множество $\Theta \subset \widetilde{Z}$ продолженных матричных функций $B_{i}(a)$ называется $\Theta$-дивизором Мальгранжса ${ }^{8}(\Theta$ зависит от начальных условий $\left.B_{i}\left(a^{0}\right)=B_{i}^{0}\right)$. Ввиду следствия 2 существует функция $\tau$, голоморфная на всем пространстве $\widetilde{Z}$, множество нулей которой совпадает с $\Theta$. Это глобальная $\tau$-функция уравнения Шлезингера. По теореме Мивы [31], [11] (см. также [2; лекция 17]) имеет место соотношение

$$
d \ln \tau(t)=\frac{1}{2} \sum_{i=1}^{n} \sum_{j=1, j \neq i}^{n} \frac{\operatorname{tr}\left(B_{i}(t) B_{j}(t)\right)}{a_{i}(t)-a_{j}(t)} d\left(a_{i}(t)-a_{j}(t)\right) .
$$

Если мы рассмотрим фуксову систему (3) как уравнение горизонтальных сечений логарифмической связности $\nabla^{0}$ (с особенностями $a_{1}^{0}, \ldots, a_{n}^{0}$ ) в голоморфно тривиальном векторном расслоении $E^{0}$ ранга $p$ над $\overline{\mathbb{C}}$, то интегрируемая деформация $(E, \nabla)$ пары $\left(E^{0}, \nabla^{0}\right)$, построенная в теореме 3 (с пространством деформации $T=\widetilde{Z}$ ), естественным образом может быть названа интегрируемой деформацией Шлезингера, поскольку ограничение $\left.(E, \nabla)\right|_{\overline{\mathbb{C}} \times D\left(a^{0}\right)}$ задает изомонодромную деформацию Шлезингера данной системы. Однако конструкцию из теоремы 3 также можно применить и для построения интегрируемой деформации $(E, \nabla)$ исходной пары $\left(E^{0}, \nabla^{0}\right)$, где векторное расслоение $E^{0}$ не является голоморфно тривиальным. Если $\operatorname{deg} E^{0}=0$, то возникает вопрос, являются ли голоморфно тривиальными расслоения $E_{t}=\left.E\right|_{\overline{\mathbb{C}} \times\{t\}}$ для почти всех значений параметра $t \in T$. Данный вопрос следует задавать в случае, когда представление монодромии связности $\nabla^{0}$ неприводимо, поскольку в противном случае имеются примеры представлений, которые не реализуются фуксовой системой ни при каком положении полюсов (см. [2; пример 11.1], [32]).

Пусть $\nabla^{0}$ - логарифмическая связность в (нетривиальном) голоморфном векторном расслоении $E^{0}$ ранга $p$ и степени нуль над $\overline{\mathbb{C}}$ такая, что ее представление монодромии неприводимо. Рассмотрим интегрируемую деформацию Шлезингера $(E, \nabla)$ пары $\left(E^{0}, \nabla^{0}\right)$, происходящую из построений теоремы 3.

Вопрос. Является ли множество

$$
\Theta=\left\{t \in T\left|E_{t}=E\right|_{\overline{\mathbb{C}} \times\{t\}} \text { не тривиально }\right\}
$$

аналитическим подмножеством коразмерности один (если оно не пусто)?

Известно, что ответ положителен в частном двумерном случае $(p=2)$, см. [33] или [34].

\footnotetext{
7 Дифференциальное уравнение имеет подвижные особенности, если особые точки его решений заполняют некоторую область в пространстве независимой переменной. Точки этой области называются подвижными особыми точками уравнения. Другими словами, подвижные особенности - те, расположение которых зависит от начальных условий уравнения. Например, особые точки $z=c$ решений $y=1 /(c-z)$ уравнения $d y / d z=y^{2}$ заполняют всю комплексную плоскость $\mathbb{C}$.

83десь термин "дивизор" не совсем точен; традиционное определение дивизора приведено несколько позже.
} 
Напомним теперь определение дивизора мероморфной функции $f: T \rightarrow \mathbb{C}$. Обозначим через $A=N \cup P$ объединение множества $N$ ее нулей и полярного множества $P$. Всякая регулярная точка $t^{0}$ множества $A$ может принадлежать только одной неприводимой компоненте множества $N$ или множества $P$. Таким образом, определен порядок этой компоненты как степень (взятая со знаком “+", если $t^{0} \in N$, и со знаком минус "-", если $t^{0} \in P$ ) соответствующего сомножителя в разложении функции $\varphi$ или $\psi$ на неприводимые множители (здесь функции $\varphi$ и $\psi$ локально определяют соответственно множества $N$ и $P$ ). Тогда дивизором мероморфной функции $f$ называют пару $(A, \kappa)$, где $\kappa=\kappa(t)-$ целочисленная функция на множестве $A^{0}$ регулярных точек множества $A$ (принимающая постоянное значение на каждой неприводимой компоненте множества $A^{0}$, это значение равно порядку компоненты). Пара $(P, \kappa)$ называется полярным дивизором мероморфной функции $f$. Через $(f)_{\infty}$ будем обозначать ограничение функции $\kappa$ на регулярные точки множества $P$.

ОБозначеНИЕ. Пусть $P$ - полярное множество функции $f$ и $t^{0} \in P$ - произвольная точка этого множества. Обозначим через $\Sigma_{t^{0}}(f)$ сумму порядков всех неприводимых компонент множества $P \cap D\left(t^{0}\right)$ (т. е. неприводимых компонент множества $P$, проходящих через точку $\left.t^{0}\right)$.

В частном (и важном для приложений) случае, представленном ниже, можно описать поведение общего решения уравнения Шлезингера в окрестности $\Theta$-дивизора.

Теорема 12 (А. А. Болибрух [35], [2; теорема 16.1]). Рассмотрим двумерное $(p=2)$ изомонодромное семейство Шлезингера (14), монодромия которо-

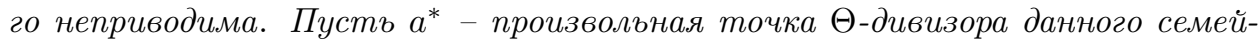
ства и

$$
E_{a^{*}}=\left.E\right|_{\overline{\mathbb{C}} \times\left\{a^{*}\right\}} \cong \mathscr{O}(k) \oplus \mathscr{O}(-k) .
$$

Тогда $\Sigma_{a^{*}}\left(B_{i}\right) \geqslant-2 k, i=1, \ldots, n$.

ЗАмЕчАниЕ 6. В работе [35] и книге [2] эта теорема сформулирована и доказана для частного случая $k=1$, однако, используя технику работы [36], можно рассмотреть и общий случай (см. [34]).

Как известно (см. [2; теорема 11.1]), имеет место неравенство $2 k \leqslant n-2$. Следовательно, оценка из теоремы 12 может быть представлена в виде $\Sigma_{a^{*}}\left(B_{i}\right) \geqslant$ $2-n$, при этом $\Sigma_{a^{*}}\left(B_{i}\right) \geqslant 3-n$ в случае нечетного $n$.

Некоторое описание (впрочем, не такое явное, как в теореме 12) локального поведения решений уравнения Шлезингера в окрестности $\Theta$-дивизора Мальгранжа имеется и в случае произвольной размерности $p$ и произвольной монодромии семейства - см. [37].

Следует заметить, что свойство Пенлеве может нарушаться в общем случае нешлезингеровской изомонодромной деформации фуксовой системы. Здесь мы вернемся к примеру 1, для того чтобы проиллюстрировать это явление (см. также работу [22] и ссылки в ней, где примеры основаны на теории голоморфных семейств фуксовых систем, фундаментальные матрицы решений которых являются рациональными по $z$ матрицами общего положения). 
ПРимеР 5. На основе примера 1 рассмотрим изомонодромное семейство фуксовых систем

$$
\begin{aligned}
\frac{d y}{d z}= & \left.\left(\begin{array}{ll}
1 & 1 \\
0 & 0
\end{array}\right) \frac{1}{z-a_{1}}+\left(\begin{array}{cc}
0 & -1 \\
0 & -1
\end{array}\right)\right) \frac{1}{z-a_{2}} \\
& \left.+\left(\begin{array}{cc}
-1 & w \\
0 & 0
\end{array}\right) \frac{1}{z-a_{3}}+\left(\begin{array}{cc}
0 & -w \\
0 & 1
\end{array}\right) \frac{1}{z-a_{4}}\right) y
\end{aligned}
$$

зависящее от параметров

$$
a=\left(a_{1}, a_{2}, a_{3}, a_{4}\right) \in \mathbb{C}^{4} \backslash \bigcup_{i \neq j}\left\{a_{i}=a_{j}\right\}, \quad w \in \mathbb{P}^{1}(\mathbb{C}) \backslash\{(-B: A)\}
$$

(см. пример 1). Напомним, что при любом фиксированном $а$ разные значения параметра $w$ определяют существенно различные фуксовы системы.

1) Зафиксируем значение $w=w^{0}$ и рассмотрим семейство $(24)$, зависящее только от параметра $a$. Это изомонодромное семейство не является ни шлезингеровским (поскольку матрицы-вычеты $B_{i}^{0}$ постоянны, но не коммутируют), ни ненормализованным шлезингеровским, поскольку не может быть преобразовано в шлезингеровское посредством замены $y^{\prime}=C^{-1}(a) y$. Действительно, последнее означало бы, что матрицы $C^{-1}(a) B_{i}^{0} C(a)$ удовлетворяют уравнению Шлезингера или, что эквивалентно, матричная дифференциальная 1-форма $\Psi=(d C) C^{-1}$ удовлетворяет системе линейных алгебраических уравнений

$$
\left[B_{i}^{0}, \Psi\right]=-\sum_{j=1, j \neq i}^{4} \frac{\left[B_{i}^{0}, B_{j}^{0}\right]}{a_{i}-a_{j}} d\left(a_{i}-a_{j}\right), \quad i=1, \ldots, 4 .
$$

Однако данная система переопределена и, как несложно проверить, не имеет решения.

Тем не менее, построенное нешлезингеровское изомонодромное семейство очевидно удовлетворяет свойству Пенлеве.

2) На пространстве $\mathbb{C}^{4} \backslash \bigcup_{i \neq j}\left\{a_{i}=a_{j}\right\}$ возьмем произвольную голоморфную функцию $f$ и рассмотрим изомонодромное семейство $(24)$, где $w=w(a)=$ $e^{1 /(f(a)-c)}-$ функция параметра $a$ и $c=c\left(a^{0}, w^{0}\right)$ - постоянная, определяемая начальным условием $w\left(a^{0}\right)=w^{0}$. Тогда гиперповерхности $\{f(a)=c\}$ образуют множество подвижных особенностей для изомонодромных деформаций исходной системы из (24). Таким образом, эти нешлезингеровские деформации не обладают свойством Пенлеве.

3) Рассматривая $a$ и $w$ как независимые параметры, получаем (24) в качестве примера изомонодромного семейства общего вида (13). Можно также найти матричную дифференциальную 1-форму $\Omega$ вида (20), соответствующую данному семейству, с резонансами $r_{j}$, равными единице, что мы оставляем читателю.

Следует также заметить, что особенности изомонодромного семейства Шлезингера - это подвижные особенности уравнения Шлезингера и только они, в то время как нешлезингеровские изомонодромные семейства могут быть не определены даже в тех точках, где матрицы-вычеты голоморфны. В данном примере - это точки $\{(a, w) \mid A w+B=0\}$, в которых изменяется монодромия. 
4.4. Приложение к системам Гарнье. Система Гарнье $\mathscr{G}_{n}(\theta)$, зависящая от $n+3$ комплексных параметров $\theta_{1}, \ldots, \theta_{n+2}, \theta_{\infty}$, была получена Р. Гарнье [38] как вполне интегрируемая система нелинейных дифференциальных уравнений в частных производных второго порядка. Впоследствии она была записана K. Окамото [39] в эквивалентной гамильтоновой форме (см. пример 2)

$$
\frac{\partial u_{i}}{\partial a_{j}}=\frac{\partial H_{j}}{\partial v_{i}}, \quad \frac{\partial v_{i}}{\partial a_{j}}=-\frac{\partial H_{j}}{\partial u_{i}}, \quad i, j=1, \ldots, n,
$$

с гамильтонианами $H_{j}=H_{j}(a, u, v, \theta)$, рационально зависящими от независимой переменной $a=\left(a_{1}, \ldots, a_{n}\right)$, неизвестных $u=\left(u_{1}, \ldots, u_{n}\right), v=\left(v_{1}, \ldots, v_{n}\right)$ и параметра $\theta=\left(\theta_{1}, \ldots, \theta_{n+2}, \theta_{\infty}\right)$.

В случае $n=1$ система Гарнье $\mathscr{G}_{1}\left(\theta_{1}, \theta_{2}, \theta_{3}, \theta_{\infty}\right)$ представляет собой эквивалентную (гамильтонову) форму шестого уравнения Пенлеве $\mathrm{P}_{\mathrm{VI}}(\alpha, \beta, \gamma, \delta)$, где

$$
\alpha=\frac{1}{2} \theta_{\infty}^{2}, \quad \beta=-\frac{1}{2} \theta_{2}^{2}, \quad \gamma=\frac{1}{2} \theta_{3}^{2}, \quad \delta=\frac{1}{2}\left(1-\theta_{1}^{2}\right) .
$$

Хорошо известно, что уравнения Пенлеве обладают свойством Пенлеве.

При $n>1$ система Гарнье, вообще говоря, не удовлетворяет свойству Пенлеве. Однако, согласно теореме Гарнье, элементарные симметрические многочлены $\sigma_{i}\left(u_{1}(a), \ldots, u_{n}(a)\right)$ от локальных решений системы Гарнье продолжаются до мероморфных функций $F_{i}$ на универсальном накрытии $\widetilde{Z}^{\prime}$ пространства $Z^{\prime}=(\mathbb{C} \backslash\{0,1\})^{n} \backslash \bigcup_{i \neq j}\left\{a_{i}=a_{j}\right\}$. Можно изучить поведение функций $F_{i}$ в окрестности их полярных множеств, используя связь между изомонодромными деформациями Шлезингера и системами Гарнье, о которой мы напоминаем далее.

Рассмотрим двумерное изомонодромное семейство Шлезингера

$$
\frac{d y}{d z}=\left(\sum_{i=1}^{n+2} \frac{B_{i}(a)}{z-a_{i}}\right) y, \quad B_{i}\left(a^{0}\right)=B_{i}^{0} \in \operatorname{sl}(2, \mathbb{C}),
$$

фуксовых систем с особыми точками $a_{1}, \ldots, a_{n}, a_{n+1}=0, a_{n+2}=1, a_{n+3}=\infty$, голоморфно зависящее от параметра $a=\left(a_{1}, \ldots, a_{n}\right) \in D\left(a^{0}\right)$, где $D\left(a^{0}\right)$ диск малого радиуса с центром в точке $a^{0}=\left(a_{1}^{0}, \ldots, a_{n}^{0}\right)$ пространства $Z^{\prime}$. Обозначим через $\pm \beta_{i}$ собственные значения матриц $B_{i}(a)$ соответственно. Напомним, что изомонодромная деформация сохраняет собственные значения матриц-вычетов $B_{i}(a)$. Из уравнения Шлезингера следует, что вычет в бесконечности является постоянной матрицей. Предположим, что эта матрица диагонализируема, т. е. $\sum_{i=1}^{n+2} B_{i}(a)=-B_{\infty}=\operatorname{diag}\left(-\beta_{\infty}, \beta_{\infty}\right)$.

Согласно теореме Мальгранжа матричные функции

$$
B_{i}(a)=\left(\begin{array}{ll}
b_{i}^{11}(a) & b_{i}^{12}(a) \\
b_{i}^{21}(a) & b_{i}^{22}(a)
\end{array}\right)
$$

могут быть продолжены на универсальное накрытие $\widetilde{Z}^{\prime}$ пространства $Z^{\prime}$ как мероморфные функции (голоморфные вне аналитического подмножества $\Theta$ коразмерности один). 

Поскольку верхний правый элемент $-\sum_{i=1}^{n+2} b_{i}^{12}(a)$ матрицы $B_{\infty}$ равен нулю,
функция

$$
P_{n}(z, a)=\left(z-a_{1}\right) \cdots\left(z-a_{n+2}\right) \sum_{i=1}^{n+2} \frac{b_{i}^{12}(a)}{z-a_{i}}
$$

при каждом фиксированном $а$ является многочленом степени $n$ по $z$. Обозначим через $u_{1}(a), \ldots, u_{n}(a)$ корни этого многочлена и определим функции $v_{1}(a), \ldots, v_{n}(a)$ :

$$
v_{j}(a)=\sum_{i=1}^{n+2} \frac{b_{i}^{11}(a)+\beta_{i}}{u_{j}(a)-a_{i}}, \quad j=1, \ldots, n
$$

Тогда имеет место следующее утверждение: $n a p a(u, v)=\left(u_{1}, \ldots, u_{n}, v_{1}, \ldots, v_{n}\right)$ удовлетворяет системе Гарнъе (25) с параметрами $2 \beta_{1}, \ldots, 2 \beta_{n+2}, 2 \beta_{\infty}-1$ (см. доказательство предложения 3.1 из [39] или [4; следствие 6.2.2, с. 207]).

Поскольку коэффициенты многочлена $P_{n}(z, a)$ выражаются рационально через верхние правые элементы $b_{i}^{12}(a)$ матриц $B_{i}(a)$, так же выражаются и элементарные симметрические многочлены от корней многочлена $P_{n}(z, a)$. Наряду с формулами для перехода от двумерного изомонодромного семейства Шлезингера $\mathrm{c} \mathrm{sl}(2, \mathbb{C})$-вычетами к системе Гарнье, существуют и формулы для обратного перехода (см. [39; предложение 3.2]). Поэтому теорему Гарнье (утверждающую, что элементарные симметрические многочлены $F_{i}=\sigma_{i}\left(u_{1}, \ldots, u_{n}\right)$ от решений системы Гарнье мероморфны на $\left.\widetilde{Z}^{\prime}\right)$ можно рассматривать как следствие теоремы Мальгранжа (теоремы 11). Кроме того, аккуратно применив теорему 12, получим приведенное ниже дополнение.

Под линейной монодромией решения системы Гарнье будем подразумевать монодромию соответствующего двумерного изомонодромного семейства Шлезингера.

Теорема 13. Пусть $(u(a), v(a))$ - решение системы Гарнъе (25), линейная монодромия которого неприводима, и пусть $\Delta_{i} \subset \widetilde{Z}^{\prime}$ обозначает полярное множество функиии $F_{i}, i=1, \ldots, n$. Тогда

а) если $\theta_{\infty}=0$ u $u_{i} \not \equiv u_{j}$ при $i \neq j$, то $\Sigma_{a^{*}}\left(F_{i}\right) \geqslant-n-1$ для всех $a^{*} \in \Delta_{i}$;

б) если $\theta_{\infty} \neq 0$, то $\Sigma_{a^{*}}\left(F_{i}\right) \geqslant-n$ для всех $a^{*} \in \Delta_{i}$, за исключением, быть может, некоторого подмножества $\Delta^{0} \subset \Delta_{i}$ положительной коразмерности; во всяком случае, $\left(F_{i}\right)_{\infty} \geqslant-n$.

ДокАЗАтельство. Рассмотрим семейство (26) с неприводимой монодромией, которое соответствует данному решению, и многочлен $P_{n}(z, a)$, построенный по матрицам-вычетам $B_{i}(a)$ (см. (27)). Его коэффициенты могут быть выражены следующим образом.

Рассмотрим элементарные симметрические многочлены

$$
\sigma_{1}(a)=\sum_{i=1}^{n+2} a_{i}, \quad \sigma_{2}(a)=\sum_{1 \leqslant i<j \leqslant n+2} a_{i} a_{j}, \quad \ldots, \quad \sigma_{n+1}(a)=a_{1} \cdots a_{n}
$$


от $a_{1}, \ldots, a_{n}, a_{n+1}=0, a_{n+2}=1$ и многочлен $Q(z)=\prod_{i=1}^{n+2}\left(z-a_{i}\right)$. Тогда

$$
P_{n}(z, a)=\sum_{i=1}^{n+2} b_{i}^{12}(a) \frac{Q(z)}{z-a_{i}}=: b(a) z^{n}+f_{1}(a) z^{n-1}+\cdots+f_{n}(a)
$$

(напомним, что $\left.\sum_{i=1}^{n+2} b_{i}^{12}(a)=0\right)$. По теореме Виета имеем

$$
\begin{aligned}
b(a) & =\sum_{i=1}^{n+2} b_{i}^{12}(a)\left(-\sigma_{1}(a)+a_{i}\right)=\sum_{i=1}^{n+2} b_{i}^{12}(a) a_{i}=\sum_{i=1}^{n} b_{i}^{12}(a) a_{i}+b_{n+2}^{12}(a), \\
f_{1}(a) & =\sum_{i=1}^{n+2} b_{i}^{12}(a)\left(\sigma_{2}(a)-\sum_{j=1, j \neq i}^{n+2} a_{i} a_{j}\right)=-\sum_{1 \leqslant i<j \leqslant n+2}\left(b_{i}^{12}(a)+b_{j}^{12}(a)\right) a_{i} a_{j} .
\end{aligned}
$$

Аналогично,

$$
f_{k}(a)=(-1)^{k} \sum_{1 \leqslant i_{1}<\cdots<i_{k+1} \leqslant n+2}\left(b_{i_{1}}^{12}(a)+\cdots+b_{i_{k+1}}^{12}(a)\right) a_{i_{1}} \cdots a_{i_{k+1}}
$$

для каждого $k=1, \ldots, n$.

Далее, по теореме Виета имеем $F_{i}(a)=(-1)^{i} f_{i}(a) / b(a)$. Согласно теореме 12 и замечанию 6 , для каждой функции $f_{i}$ и любой точки $a^{*}$, принадлежащей $\Theta$-дивизору семейства (26), справедлива оценка $\Sigma_{a^{*}}\left(f_{i}\right) \geqslant-n-1$.

Несложно проверить (используя уравнение Шлезингера), что $d b(a)=-\theta_{\infty} \times$ $\sum_{i=1}^{n} b_{i}^{12}(a) d a_{i}$, где $\theta_{\infty}=2 \beta_{\infty}-1$.

а) Если $\theta_{\infty}=0$, то $d b(a) \equiv 0$, следовательно, $b(a) \equiv$ const $\neq 0$. В самом деле, если $b(a) \equiv 0$, то $P_{n}(z, a)$ - многочлен степени $n-1$ по $z$, и $u_{i}(a) \equiv u_{j}(a)$ для некоторых $i \neq j$, а это противоречит условиям теоремы. Таким образом, $\Sigma_{a^{*}}\left(F_{i}\right)=\Sigma_{a^{*}}\left(f_{i}\right) \geqslant-n-1$ в этом случае.

б) Если $\theta_{\infty} \neq 0$, то

$$
\begin{gathered}
b_{i}^{12}(a)=-\frac{1}{\theta_{\infty}} \frac{\partial b(a)}{\partial a_{i}}, \quad i=1, \ldots, n \\
b_{n+2}^{12}(a)=b(a)-\sum_{i=1}^{n} b_{i}^{12}(a) a_{i}, \quad b_{n+1}^{12}(a)=-b_{n+2}^{12}(a)-\sum_{i=1}^{n} b_{i}^{12}(a) .
\end{gathered}
$$

Следовательно, если функция $b$ голоморфна в точке $a^{\prime} \in \widetilde{Z}^{\prime}$, то голоморфны и функции $b_{i}^{12}, i=1, \ldots, n+2$, а значит, и функции $f_{i}$. Поэтому точки $a^{*} \in \Delta_{i}$ могут быть двух типов: такие, что $b\left(a^{*}\right)=0$ (и тогда $\Sigma_{a^{*}}\left(F_{i}\right) \geqslant-1$, поскольку функция $b$ неприводима $\left.{ }^{9}\right)$, или такие, которые принадлежат полярному множеству $\Delta \subset \Theta$ функции $b$.

9 Действительно, если $d b\left(a^{\prime}\right) \equiv 0$ для некоторой точки $a^{\prime} \in\{b(a)=0\}$, то $\sum_{i=1}^{n} b_{i}^{12}\left(a^{\prime}\right) d a_{i} \equiv 0$ и $b_{1}^{12}\left(a^{\prime}\right)=\cdots=b_{n}^{12}\left(a^{\prime}\right)=0$. Принимая во внимание соотношения $(28)$, получаем также, что $b_{n+2}^{12}\left(a^{\prime}\right)=0$ и $b_{n+1}^{12}\left(a^{\prime}\right)=0$. Но это противоречит неприводимости монодромии семейства (26). 
Обозначим через $\Delta^{0} \subset \Delta$ множество точек неопределенности функции $b$. Тогда в окрестности любой точки $a^{*} \in \Delta \backslash \Delta^{0}$ она может быть представлена в виде

$$
b(a)=\frac{h(a)}{\tau_{1}^{j_{1}}(a) \cdots \tau_{r}^{j_{r}}(a)}, \quad j_{1} \geqslant 1, \ldots, j_{r} \geqslant 1,
$$

где $\tau_{l}, h$ - голоморфные возле точки $a^{*}$ функции, $h\left(a^{*}\right) \neq 0$ и при этом функции $\tau_{l}$ неприводимы в точке $a^{*}$, в то время как

$$
f_{i}(a)=\frac{g_{i}(a)}{\tau_{1}^{k_{1}}(a) \cdots \tau_{r}^{k_{r}}(a)}, \quad k_{1}+\cdots+k_{r} \leqslant n+1,
$$

где $g_{i}$ - голоморфная возле точки $a^{*}$ функция $(i=1, \ldots, n)$. Таким образом,

$$
\frac{f_{i}(a)}{b(a)}=\frac{g_{i}(a)}{\tau_{1}^{k_{1}}(a) \cdots \tau_{r}^{k_{r}}(a)} / \frac{h(a)}{\tau_{1}^{j_{1}}(a) \cdots \tau_{r}^{j_{r}}(a)}=\frac{g_{i}(a) / h(a)}{\tau_{1}^{k_{1}-j_{1}}(a) \cdots \tau_{r}^{k_{r}-j_{r}}(a)},
$$

поэтому

$$
\Sigma_{a^{*}}\left(F_{i}\right)=-\sum_{\alpha}\left(k_{\alpha}-j_{\alpha}\right) \geqslant-n
$$

(суммирование производится по таким индексам $\alpha$, для которых $k_{\alpha}-j_{\alpha}>0$ ), что доказывает первую часть утверждения б).

В окрестности точки $a^{*} \in \Delta^{0}$ разложения (29), (30) для функций $b, f_{i}$ соответственно также имеют место, но только $h\left(a^{*}\right)=0$. Однако, ввиду неприводимости функции $b$, все неприводимые сомножители в разложении $h(a)=$ $h_{1}(a) \cdots h_{s}(a)$ возле точки $a^{*}$ различны (можно также считать, что ни одна из функций $h_{i}$ не совпадает ни с какой функцией $\left.\tau_{l}\right)$. Поскольку $k_{l}-j_{l} \leqslant n$ для всех $l=1, \ldots, r$, то вторая часть утверждения б) следует из разложения

$$
\frac{f_{i}(a)}{b(a)}=\frac{g_{i}(a)}{h_{1}(a) \cdots h_{s}(a) \tau_{1}^{k_{1}-j_{1}}(a) \cdots \tau_{r}^{k_{r}-j_{r}}(a)} .
$$

Теорема доказана.

ЗАмЕчАнИЕ 7. Из замечания 6 следует, что в случае четного $n$ во всех оценках из теоремы 13 можно заменить $n$ на $n-1$.

В частности, полярные множества функций $F_{1}(a)=u_{1}(a)+u_{2}(a)$ и $F_{2}(a)=$ $u_{1}(a) u_{2}(a)$, где $\left(u_{1}, u_{2}, v_{1}, v_{2}\right)$ - решение системы Гарнье $\mathscr{G}_{2}\left(\theta_{1}, \ldots, \theta_{4}, \theta_{\infty}\right)$, соответствующее двумерному изомонодромному семейству Шлезингера с пятью особыми точками и неприводимой монодромией, являются аналитическими подмногообразиями, и $\left(F_{i}\right)_{\infty} \geqslant-2$. (Заметим, что расслоение $E_{a^{*}}$, соответствующее точке $a^{*} \Theta$-дивизора данного семейства, имеет вид $E_{a^{*}} \cong \mathscr{O}(1) \oplus \mathscr{O}(-1)$, откуда следует регулярность $\Theta$-дивизора; см. [2; теорема 16.2] или [34].)

\section{Приложение А. О результатах п. 3.3}

Здесь мы рассмотрим общий случай семейств (13) фуксовых систем и приведем интерпретацию результатов п. 3.3, основанную на использовании пространств модулей. Мы изложим лишь общую схему альтернативного доказательства представления (20). Подробное изложение можно найти в работе [23]. 
Для построения используется техника преобразований Шлезингера - калибровочных преобразований матричной дифференциальной 1-формы $\omega^{0}$, pacсматриваемой как форма связности голоморфно тривиального векторного расслоения над $\overline{\mathbb{C}}$. Такой подход широко используется в работах по проблеме Римана-Гильберта и связанным с ней задачам. Указанные калибровочные преобразования сохраняют монодромию системы, изменяя ее показатели. Ввиду того что сумма вычетов 1-формы является инвариантом, всякое изменение показателя должно быть компенсировано еще одним изменением в той же или другой особой точке. Таким образом, простейшее возможное преобразование Шлезингера, в дальнейшем - элементарное преобразование, повышает на единицу одно из нормирований в некоторой особой точке и понижает также на единицу одно нормирование в какой-то из точек. Описанные преобразования легко строятся в явном виде (подробнее см. в [23]).

Возьмем теперь исходную резонансную фуксову систему. Заметим, что последовательным применением элементарных преобразований мы можем разрешить все резонансы системы, добавив к ней вспомогательную точку $a^{*}$. В самом деле, элементарное преобразование существенно затрагивает не более двух особых точек, следовательно, компенсируя каждое нужное нам изменение в нормированиях соответствующим изменением показателей в точке $a^{*}$, действительно можно избавиться от всех резонансов. Для полученного семейства, имеющего, быть может, единственную резонансную точку $a^{*}$, существует шлезингеровское изомонодромное семейство.

Заметим, что описанные калибровки фуксовых систем могут быть обобщены до преобразований, действующих на изомонодромные семейства. Ввиду их явного вида и довольно простой структуры действие таких и обратных преобразований на шлезингеровскую 1-форму может быть вычислено точно. Важное наблюдение теперь показывает, что в процессе обратного перехода от шлезингеровского семейства к исходному встречаются два разных шага: рождение резонанса и его усиление. Создавая резонанс, выбор лидирующего направления из собственного подпространства монодромии следует производить в соответствии с данными нашей исходной системы. Освобождая же параметры $\left\{w_{1}, \ldots, w_{d}\right\}$, задающие эти направления, мы получаем целиком связную компоненту $\mathscr{W}$ пространства модулей фуксовых систем - множество всех возможных фуксовых систем с заданной монодромией и показателями. Остается теперь добавить эти параметры в калибровочные преобразования в качестве новых дополнительных переменных и поправить калибровочное действие, добавив дифференцирование по этим параметрам. Так как эти новые переменные однозначно выделяют фуксову систему из всех систем, имеющих те же монодромию и особенности, то любое изомонодромное семейство может быть получено калибровочными преобразованиями из шлезингеровского семейства. Это показывает также независимость конструкции от положения точки $a^{*}$. Ее положение выражается через добавленные переменные и является несущественным. Прямое вычисление дает представление (20).

Важным наблюдением является то, что с помощью представления всякого изомонодромного семейства как результата элементарных калибровочных преобразований шлезингеровского семейства можно оценить рост его коэффи- 
циентов в ходе деформации, а также проиллюстрировать возможное наличие или нарушение свойства Пенлеве для уравнений деформации. Действительно, предположение о том, что новые переменные $w_{i}$ являются функциями параметров $a_{1}, \ldots, a_{n}$, приводит 1-форму $\Omega$ к виду $(19)$, и если эти функции имеют более чем степенной рост, очевидным образом получается нарушение свойства Пенлеве для итогового семейства (как мы видели в примере 5). Более того, это является единственной возможной конструкцией нарушения свойства Пенлеве для изомонодромных деформаций (14) фуксовых систем. С другой стороны, изучение точного вида калибровочных преобразований, использованных в вышеприведенных рассуждениях, может гарантировать наличие свойства Пенлеве для итогового семейства, если не будет зависимости вида $w=w(a)$ или если функции $w_{i}(a)$ имеют соответствующий рост.

\section{Приложение В. Симплектические структуры}

В этом приложении мы попробуем осветить геометрию, лежащую в основе изомонодромных деформаций, и дадим краткий обзор связей таких деформаций с симплектическими структурами на пространствах модулей связностей и представлений.

Начнем с фуксовых систем. Отправной точкой здесь является изучение свойств отображения монодромии. Это отображение, являясь крайне трансцендентным, обладает в то же время рядом замечательных свойств. Взяв отображение, сопоставляющее мероморфному дифференциальному уравнению его данные монодромии, можно расширить его до отображения (соответствующим образом определенного) пространства модулей мероморфных связностей, принимающего значения в пространстве модулей представлений фундаментальной группы. Пространство модулей связностей естественным образом расслоено над пространством параметров деформации, пространству модулей представлений также естественным образом сопоставляется некоторое расслоение над той же базой. Более подробно можно сказать, что это расслоение получается расширением пространства модулей путем добавления дополнительных данных, а именно положений проколов, обходимых образующими фундаментальной группы. Очевидным образом отображение монодромии легко определить и между этими двумя расслоениями. Оба расслоения оказываются оснащенными естественными симплектическими структурами, а отображение монодромии задает симплектоморфизм. Естественная плоская связность Эресмана в расслоении, соответствующем представлениям, имеет очевидное описание: горизонтальными являются сечения, проходящие над каждой точкой через одно и то же представление. Обратным образом этих сечений относительно отображения монодромии будут изомонодромные семейства фуксовых систем. Таким образом, уравнения изомонодромных деформаций описывают естественную плоскую симплектическую связность на пространстве модулей фуксовых систем, расслоенном по положению особых точек системы. Записанная в координатах форма связности варьируется от (15) в простейшем случае до (20) в общем виде. 
Этот подход был обобщен Ф. Боалчем [40]. Им дано описание пространств модулей, симплектических (в общем случае пуассоновых) структур и соответствующих отображений для систем с особыми точками положительного ранга Пуанкаре (нефуксовыми особенностями). Он также показал, что общая симплектическая геометрия лежит в основе и таких обобщенных деформаций: отображение монодромии остается симплектоморфизмом между соответствующим образом определенными пространствами модулей.

\section{Список литературы}

[1] О. Форстер, Римановы поверхности, Мир, М., 1980, 248 с.; пер. с нем.: O. Forster, Riemannsche Flächen, Heidelberger Taschenbucher, 184, Springer-Verlag, Berlin-New York, 1977, x+223 pp.

[2] А.А. Болибрух, Обратные задачи монодромии в аналитической теории дифференциальных уравнений, МЦНМО, М., 2009, ISBN: 978-5-94057-510-8, 220 с., http://biblio.mccme.ru/node/2154.

[3] A. H. M. Levelt, "Hypergeometric functions. II", Nederl. Akad. Wetensch. Proc. Ser. A, 64 (1961), 373-385.

[4] K. Iwasaki, H. Kimura, S. Shimomura, M. Yoshida, From Gauss to Painlevé. A modern theory of special functions, Aspects Math., E16, Friedr. Vieweg, Braunschweig, 1991, ISBN: 3-528-06355-6, xii+347 pp.

[5] Ф. Уорнер, Основы теории гладких многообразий и групп Ли, Мир, М., 1987, 304 с.; пер. с англ.: F. W. Warner, Foundations of differentiable manifolds and Lie groups, Corrected reprint of the 1971 edition, Grad. Texts in Math., 94, SpringerVerlag, New York-Berlin, 1983, ISBN: 0-387-90894-3, ix+272 pp.

[6] S. Kobayashi, Differential geometry of complex vector bundles, Publ. Math. Soc. Japan, 15, Princeton Univ. Press, Princeton, NJ; Iwanami Shoten, Tokyo, 1987, ISBN: 0-691-08467-X, xii+305 pp.

[7] Р. С. Ганнинг, Х. Росси, Аналитические функции многих комплексных переменных, Мир, М., 1969, 395 с.; пер. с англ.: R. C. Gunning, H. Rossi, Analytic functions of several complex variables, Prentice-Hall, Englewood Cliffs, NJ, 1965, xiv+317 pp.

[8] А. Т. Фоменко, Д. Б. Фукс, Курс гомотопической топологии, Наука, М., 1989, ISBN: 5-02-013929-7, 496 c.

[9] K. Stein, "Überlagerungen holomorph-vollständiger komplexer Räume", Arch. Math. (Basel), 7:5 (1956), 354-361.

[10] H. Grauert, "Ein Theorem der analytischen Garbentheorie und die Modulräume komplexer Strukturen", Inst. Hautes Études Sci. Publ. Math., 5 (1960), 233-292, http://www.numdam.org/item?id=PMIHES_1960__5_-5_0.

[11] M. Jimbo, T. Miwa, K. Ueno, "Monodromy preserving deformations of linear differential equations with rational coefficients. I. General theory and $\tau$-function", Phys. D, 2:2 (1981), 306-352.

[12] B. Malgrange, "Sur les déformations isomonodromiques. II. Singularités irrégulières", Progr. Math., 37, Birkhäuser Boston, Boston, MA, 1983, 427-438.

[13] J. Palmer, "Zeros of the Jimbo, Miwa, Ueno tau function", J. Math. Phys., 40:12 (1999), 6638-6681.

[14] M. Bertola, M. Y. Mo, "Isomonodromic deformation of resonant rational connections", IMRP Int. Math. Res. Pap., 2005, № 11, 565-635.

[15] A. A. Bolibruch, "On isomonodromic deformations of Fuchsian systems", J. Dyn. Control Syst., 3:4 (1997), 589-604. 
[16] L. Schlesinger, "Über die Lösungen gewisser linearer Differentialgleichungen als Funktionen der singulären Punkte", J. Reine Angew. Math., 129 (1905), 287-294.

[17] L. Schlesinger, "Über eine Klasse von Differentialsystem beliebiger Ordnung mit festen kritischen Punkten", J. Reine Angew. Math., 141 (1912), 96-145.

[18] M. Jimbo, T. Miwa, Y. Mori, M. Sato, "Density matrix of an impenetrable Bose gas and the fifth Painlevé transcendent", Phys. D, 1:1 (1980), 80-158.

[19] B. Malgrange, "Déformations isomonodromiques, forme de Liouville, fonction $\tau$ ", Ann. Inst. Fourier (Grenoble), 54:5 (2004), 1371-1392, http://aif.cedram.org/aif-bin/ item?id=AIF_2004__54_5_1371_0.

[20] А.А. Болибрух, "Об изомонодромных слияниях фуксовых особенностей”, Локальные и глобальные задачи теории особенностей, Сб. статей. К 60-летию со дня рождения академика Владимира Игоревича Арнольда, Тр. МИАН, 221, Наука, M., 1998, 127-142; англ. пер.: A. A. Bolibrukh, "On isomonodromic confluences of Fuchsian singularities", Proc. Steklov Inst. Math., 221 (1998), 117-132.

[21] A. V. Kitaev, "Non-Schlesinger deformations of ordinary differential equations with rational coefficients", J. Phys. A, 34:11 (2001), 2259-2272.

[22] V. Katsnelson, D. Volok, "Deformations of Fuchsian systems of linear differential equations and the Schlesinger system", Math. Phys. Anal. Geom., 9:2 (2006), 135-186.

[23] В. А. Побережный, "Общая линейная задача изомонодромной деформации фуксовых систем", Матем. заметки, 81:4 (2007), 599-613; англ. пер.: V. A. Poberezhnyi, "General linear problem of the isomonodromic deformation of Fuchsian systems", Math. Notes, 81:4 (2007), 529-542.

[24] B. Malgrange, "Sur les déformations isomonodromiques. I. Singularités régulières", Mathematics and physics (Paris, 1979/1982), Progr. Math., 37, Birkhäuser Boston, Boston, MA, 1983, 401-426.

[25] L. Boutet de Monvel, "Problème de Riemann-Hilbert. III", Mathematics and physics (Paris, 1979/1982), Progr. Math., 37, Birkhäuser Boston, Boston, MA, 1983, 299-306.

[26] M. Atiyah, K-theory, W. A. Benjamin, New York-Amsterdam, 1967, 166 pp.

[27] M. Schechter, Principles of functional analysis, Academic Press, New York-London, 1971, 383 pp.

[28] G.D. Birkhoff, "A theorem on matrices of analytic functions", Math. Ann., 74:1 (1913), 122-133.

[29] A. Grothendieck, "La théorie de Fredholm", Bull. Soc. Math. France, 84 (1956), 319-384.

[30] G. Helminck, "Deformations of connections, the Riemann-Hilbert problem and $\tau$-functions", Computational and combinatorial methods in system theory (Stockholm, 1985), North-Holland, Amsterdam, 1986, 75-89.

[31] M. Sato, T. Miwa, M. Jimbo, "Aspects of holonomic quantum fields. Isomonodromic deformation and Ising model", Complex analysis, microlocal calculus and relativistic quantum theory (Proc. Internat. Colloq., Centre Phys., Les Houches, 1979), Lect. Notes in Phys., 126, Springer, Berlin, 1980, 429-491.

[32] И.В. Вьюгин, "Фуксовы системы с вполне приводимой монодромией", Матем. заметки, 85:6 (2009), 817-825; англ. пер.: I. V. V'yugin, "Fuchsian systems with completely reducible monodromy", Math. Notes, 85:6 (2009), 780-786.

[33] V. Heu, "Stability of rank 2 vector bundles along isomonodromic deformations", Math. Ann., 344:2 (2009), 463-490.

[34] R. R. Gontsov, I. V. Vyugin, "Apparent singularities of Fuchsian equations, the Painlevé VI equation, and Garnier systems", 2009, arXiv: 0905.1436.

[35] A. A. Bolibruch, "On orders of movable poles of the Schlesinger equation", J. Dyn. Control Syst., 6:1 (2000), 57-74. 
[36] А. А. Болибрух, "О tau-функции уравнения изомонодромных деформаций Шлезингера", Матем. заметки, 74:2 (2003), 184-191; англ. пер.: А. A. Bolibrukh, "On the tau function for the Schlesinger equation of isomonodromic deformations", Math. Notes, 74:2 (2003), 177-184.

[37] Р.Р. Гонцов, "О решениях уравнения Шлезингера в окрестности $\Theta$-дивизора Мальгранжа", Матем. заметки, 83:5 (2008), 779-782; англ. пер.: R. R. Gontsov, "On solutions of the Schlesinger equation in the neigborhood of the Malgrange @-divisor", Math. Notes, 83:5 (2008), 707-711.

[38] R. Garnier, "Sur des équations différentielles du troisième ordre dont l'intégrale générale est uniforme et sur une classe d'équations nouvelles d'ordre supérieur dont l'intégrale générale a ses points critiques fixes", Ann. Sci. École Norm. Sup. (3), 29 (1912), 1-126.

[39] K. Okamoto, "Isomonodromic deformation and Painlevé equations, and the Garnier system", J. Fac. Sci. Univ. Tokyo Sect. IA Math., 33:3 (1986), 575-618.

[40] P. Boalch, "Symplectic manifolds and isomonodromic deformations", Adv. Math., 163:2 (2001), 137-201.

P. P. Гонцов (R. R. Gontsov)

Институт проблем передачи информации

им. А. А. Харкевича РАН

E-mail: rgontsov@inbox.ru

\section{B. А. Побережный (V. A. Poberezhnyi)}

Институт теоретической и экспериментальной физики им. А. И. Алиханова

E-mail: poberezh@itep.ru

\section{Г. Ф. Хельминк (G. F. Helminck)}

Korteweg-de Vries Institute of Mathematics, University of Amsterdam, The Netherlands

E-mail: g.f.helminck@uva.nl
Поступила в редакцию

07.12 .2010 